TO APPEAR IN The Astrophysical Journal.

Preprint typeset using LATEX style emulateapj v. 26/01/00

\title{
A NEW SAMPLE OF LOW-MASS BLACK HOLES IN ACTIVE GALAXIES
}

\author{
JENNY E. GREENE ${ }^{1}$ \\ Department of Astrophysical Sciences, Princeton University, Princeton, NJ \\ LUIS C. Ho \\ The Observatories of the Carnegie Institution of Washington, 813 Santa Barbara St., Pasadena, CA 91101
}

To appear in The Astrophysical Journal.

\begin{abstract}
We present an expanded sample of low-mass black holes (BHs) found in galactic nuclei. Using standard virial mass techniques to estimate BH masses, we select from the Fourth Data Release of the Sloan Digital Sky Survey all broad-line active galaxies with masses $<2 \times 10^{6} M_{\odot}$. BHs in this mass regime provide unique tests of the relationship between BHs and galaxies, since their late-type galaxy hosts do not necessarily contain classical bulges. Furthermore, they provide observational analogs of primordial seed BHs and are expected, when merging, to provide strong gravitational signals for future detectors such as LISA. From our preliminary sample of 19, we have increased the total sample by an order of magnitude to 174 , as well as an additional 55 (less secure) candidates. The sample has a median BH mass of $\left\langle M_{\mathrm{BH}}\right\rangle=1.3 \times 10^{6} M_{\odot}$, and in general the objects are radiating at high fractions of their Eddington limits. We investigate the broad spectral properties of the sample; 55 are detected by ROSAT, with soft X-ray luminosities in the range $10^{40}$ to $7 \times 10^{43} \mathrm{ergs} \mathrm{s}^{-1}$. Much like the preliminary sample, these objects are predominantly radio-quiet $\left(R \equiv f_{6 \mathrm{~cm}} / f_{4400}<10\right)$, but 11 objects are detected at $20 \mathrm{~cm}$, with radio powers $\left(10^{21}-10^{23} \mathrm{~W} \mathrm{~Hz}^{-1}\right)$ that may arise from either star formation or nuclear activity; only $1 \%$ of the sample is radio-loud. We further confirm that, with $\left\langle M_{g}\right\rangle=-19.3$ and $\langle g-r\rangle=0.7 \mathrm{mag}$, the host galaxies are low-mass, late-type systems. At least $40 \%$ show disk-like morphologies, and the combination of host galaxy colors and higher-order Balmer absorption lines indicate intermediate-age stellar populations in a subset of the sample.

Subject headings: galaxies: active — galaxies: nuclei — galaxies: Seyfert
\end{abstract}

\section{INTRODUCTION}

There are many strong observational connections between galaxy bulges and central supermassive black holes (BHs). BH masses correlate surprisingly tightly with bulge properties, including luminosity (e.g., Marconi \& Hunt 2003) and stellar velocity dispersion $\left(M_{\mathrm{BH}}-\sigma_{*}\right.$; Ferrarese \& Merritt 2000; Gebhardt et al. 2000a; Tremaine et al. 2002). Also active galactic nuclei (AGNs) in the local Universe are predominantly found in massive, bulge-dominated galaxies (Ho et al. 1997b; Kauffmann et al. 2003). Perhaps the formation of BHs and bulges are related, in which case we might not expect bulgeless galaxies to obey scaling relations between $\mathrm{BHs}$ and galaxies, or even necessarily host a central BH. Indeed, dynamical study of the bulgeless spiral galaxy M33 places strong limits on the presence of a dark central massive object (Gebhardt et al. 2001), while any $\mathrm{BH}$ in the nucleus of the dwarf spheroidal galaxy NGC 205 has been shown to lie below the low-mass extrapolation of the $M_{\mathrm{BH}}-\sigma_{*}$ relation (Valluri et al. 2005). On the other hand, the M31 globular cluster G1 shows dynamical and radiative evidence for a central BH (Gebhardt et al. 2002, 2005; Pooley \& Rappaport 2006; Ulvestad et al. 2007). However, the mixed stellar population and high degree of rotational support in this massive cluster suggest that G1 is actually the nucleus of a tidally stripped dwarf galaxy (e.g., Meylan et al. 2001). Thus, dynamical studies present an ambiguous verdict on the presence of nuclear BHs in dwarf stellar systems. Unfortunately, it is not currently feasible to spatially resolve the gravitational sphere of influence of $\mathrm{a} \sim 10^{5} M_{\odot} \mathrm{BH}$ outside the Local Group, in order to search for BHs in more dwarf stellar systems using dynamical methods.

Although they are difficult to find, the occupation fraction of nuclear BHs in dwarf systems and the space density of lowmass BHs are of considerable interest. Apart from furnishing additional insight into the possible origin of the $M_{\mathrm{BH}}-\sigma_{*}$ relation, low-mass BHs provide low-redshift counterparts to the primordial seed BHs; the low-mass cut-off in the $\mathrm{BH}$ mass function today provides a constraint on the mass function of seed BHs. The merging of BHs in this mass range is expected to provide a strong signal for the gravitational wave experiment LISA (e.g., Hughes 2002). Furthermore, gravitational radiation recoil is expected to impart velocities to $\mathrm{BH}$ merger remnants that exceed the escape velocities of dwarf galaxies (e.g., Favata et al. 2004; Merritt et al. 2004). This effect alone might decrease the occupation fraction of BHs in dwarf galaxies.

In the absence of detectable dynamical signatures, we are forced to rely on less direct evidence for the presence of nuclear BHs, namely AGN activity. In fact, there are two well-studied AGNs in dwarf galaxies: NGC 4395 is a bulgeless spiral galaxy (Filippenko \& Sargent 1989), while POX 52 (Kunth et al. 1987) is a dwarf spheroidal galaxy (Barth et al. 2004)2. BH mass determinations are far less certain in the absence of dynamical constraints, but a variety of techniques yield $\mathrm{BH}$ masses for each object of $\sim 10^{5} M_{\odot}$ (Filippenko \& Ho 2003; Barth et al. 2004; Peterson et al. 2005). Greene \& Ho (2004; GH hereafter) performed a systematic search for AGNs with

\footnotetext{
${ }^{1}$ Hubble Fellow and Princeton-Carnegie Fellow.

${ }^{2}$ Such objects are commonly referred to as dwarf elliptical galaxies in the literature. However, because their structure is quite different from that of classical elliptical galaxies, we prefer to refer to them as dwarf spheroidal systems.
} 
Table 1. The SDSS Sample

\begin{tabular}{rcccccc}
\hline \hline $\begin{array}{l}\text { ID } \\
(1)\end{array}$ & $\begin{array}{c}\text { SDSS Name } \\
(2)\end{array}$ & $\begin{array}{c}\text { Flag } \\
(3)\end{array}$ & $\begin{array}{c}z \\
(4)\end{array}$ & $\begin{array}{c}g \\
(5)\end{array}$ & $\begin{array}{c}g-r \\
(6)\end{array}$ & $\begin{array}{c}A_{g} \\
(7)\end{array}$ \\
\hline 1 & SDSS J000111.15-100155.5 & & 0.0489 & 18.6 & 0.7 & 0.21 \\
2 & SDSS J000308.47+154842.3 & & 0.118 & 18.2 & 0.5 & 0.16 \\
3 & SDSS J000605.59-092007.0 & $\mathrm{c}$ & 0.0699 & 17.9 & 0.7 & 0.34 \\
\hline
\end{tabular}

Note. - Col. (1): Identification number assigned in this paper. Col (2): Official SDSS name. Col. (3): Objects that are beneath our detection threshold are indicated with $c$. Previous identifier, if relevant, is indicated Col. (4): Redshift measured by the SDSS pipeline. Col. (5): Petrosian $g$ magnitude. Objects with questionable Petrosian magnitudes (flagged as many_petro or no_petro by the SDSS pipeline) are noted with $*$, and the model magnitudes are tabulated. Col. (6): Petrosian $g-r$ color. Col. (7): Galactic extinction in the $g$ band. Table 1 is available in its entirety via the link to the machine-readable table above. The following is included only as a guide to content and presentation.

BH masses $<10^{6} M_{\odot}$, using the First Data Release of the Sloan Digital Sky Survey (SDSS; York et al. 2000) and recovered 19 objects in that preliminary search. Remarkably, follow-up spectroscopy using ESI on Keck suggests that these sources, as well as NGC 4395, POX 52, and G1, are consistent with the lowmass extrapolation of the $M_{\mathrm{BH}}-\sigma_{*}$ relation (Barth et al. 2005). At the same time, many of the host galaxies are late-type galaxies without classical bulges (J. E. Greene, et al. in preparation). Clearly, more objects are needed to both confirm this preliminary result, and also to constrain the structural parameters of the host galaxies. To this end, we have repeated our analysis using the Fourth Data Release of the SDSS (DR4; AdelmanMcCarthy et al. 2006), and present the updated sample here.

Our goal is to study the properties of the lowest-mass BHs that are identifiable with the SDSS. The upper mass limit on this sample is somewhat arbitrary. Within the inactive sample of galaxies with dynamical BH masses, the BH in M32, $M_{\mathrm{BH}}=\left(2.5_{-2.0}^{+3.0}\right) \times 10^{6} M_{\odot}$, has the lowest mass (Tremaine et al. 2002; excluding the Milky Way, whose modern mass is $3.5 \times 10^{6} M_{\odot}$; e.g., Ghez et al. 2005; Eisenhauer et al. 2005). We have adopted $2 \times 10^{6} M_{\odot}$ as the upper limit for our search. Because dynamical methods cannot extend into this regime, we previously had constraints on neither the existence of lowermass BHs nor on whether they obey similar scaling relations with galaxy bulge properties as their high-mass cousins. Furthermore, while there are good reasons to believe that not every dwarf stellar system contains a BH, we do not know at what mass the occupation fraction departs from unity.

Throughout we assume the following cosmological parameters to calculate distances: $H_{0}=100 h=71 \mathrm{~km} \mathrm{~s}^{-1} \mathrm{Mpc}^{-1}$, $\Omega_{m}=0.27$, and $\Omega_{\Lambda}=0.75$ (Spergel et al. 2003).

\section{SAMPLE SELECTION AND ANALYSIS}

Our low-mass BHs are selected from the sample of broadline AGNs with $z<0.352$ described in detail by Greene \& Ho (2007b) and briefly reviewed here for completeness. We begin with DR4 of the SDSS and search for all AGNs with broad $\mathrm{H} \alpha$, where "broad" in this context means a significant extra component relative to the narrow-line profile (based on the [S II] $\lambda \lambda 6716,6731$ doublet; e.g., Ho et al. 1997c). Continuum subtraction, which is crucial to uncover low-contrast broad lines, is performed with the principle component analysis method developed by Hao et al. (2005). Our subsequent selec- tion technique comprises a two-step procedure: we first select objects with high root-mean-square (rms) deviations above the continuum in the region potentially containing broad $\mathrm{H} \alpha$, and then perform more detailed profile fitting to isolate those objects with broad $\mathrm{H} \alpha$ profiles. Unfortunately, this selection process results in many sources of such low $\mathrm{H} \alpha$ luminosity that (1) their nature is ambiguous and (2) our ability to recover a reliable BH mass is severely compromised. Based on simulations, we thus impose a combined rms-weighted flux and equivalent width (EW) cut designed to minimize spurious detections. The resulting sample comprises 8435 objects.

Since we cannot use stellar dynamical methods to measure $\mathrm{BH}$ masses, we instead use the photoionized broad-line region (BLR) gas as a dynamical tracer of the BH mass. While the BLR velocity dispersion is derived from the line width, the BLR radius is inferred from the AGN luminosity (in this case $\mathrm{H} \alpha$; Greene \& Ho 2005b). The so-called radius-luminosity relation is derived from reverberation mapping of $\sim 30$ AGNs, for which radii are measured based on the delay between variations in the AGN photoionizing continuum and BLR line emission (e.g., Kaspi et al. 2005; Greene \& Ho 2005b; Bentz et al. 2006). The "virial" $\mathrm{BH}$ mass is simply $M_{\mathrm{BH}}=f R v^{2} / G$, where $f$ is a scaling factor that accounts for the unknown geometry of the BLR, assumed here to be spherical ( $f=0.75$; Netzer 1990). Although BH masses derived from reverberation mapping are susceptible to large systematic errors (due to uncertainties in the BLR geometry and kinematics; e.g., Krolik 2001), remarkably they have been shown to agree with $\sigma_{*}$, in those cases for which $\sigma_{*}$ can be measured. The measured scatter is $\sim 0.3$ dex (Gebhardt et al. 2000b; Ferrarese et al. 2001; Onken et al. 2004; Nelson et al. 2004). Virial masses are less direct, since they rely on the radius-luminosity relation; in the largest such comparison to date, Greene \& Ho (2006) find a scatter of 0.4 dex of single-epoch virial masses about the $M_{\mathrm{BH}}-\sigma_{*}$ relation. Our BH mass estimator is discussed in detail in the Appendix. Here we simply note that Greene \& Ho (2007b) used an old version of the radius-luminosity relation slope, while here we have updated the value to that found by Bentz et al. (2006). In general, the derived masses increase by $\sim 0.3$ dex.

Selecting those broad-line AGNs with masses $<2 \times 10^{6} M_{\odot}$ results in a sample of 174 objects, which are the main subject of this paper (Fig. 1). In addition, some (55) of the objects below the detection threshold are nevertheless interesting candidates. These objects have been selected by manual inspection of the spectra, and thus comprise a biased and incomplete sample. We worry, as explained above, that our BH masses are not stable for these $\mathrm{H} \alpha$ luminosities, but we deem these objects worthy of follow-up spectroscopy. In what follows we will refer to these objects as the $c$ ("candidate") sample, and we will present all trends including and excluding these objects. Basic properties of the sample are summarized in Table 1.

\subsection{Fe II Fitting}

Our modeling of the $\mathrm{H} \beta$ region warrants some additional discussion. While the continua of our spectra, in general, are dominated by stellar light, in some cases there is an additional "pseudo-continuum" component contributed by broad Fe II multiplets. The Fe II emission extends over the entire optical and UV spectrum, but is especially troublesome in the wavelength ranges $4400-4800 \AA$ and 5150-5500 $\AA$. In principle, both the $\mathrm{H} \beta$ and [O III] $\lambda \lambda 4959,5007$ fits can be severely compromised when the Fe II component is ignored (see Fig. 2). We 

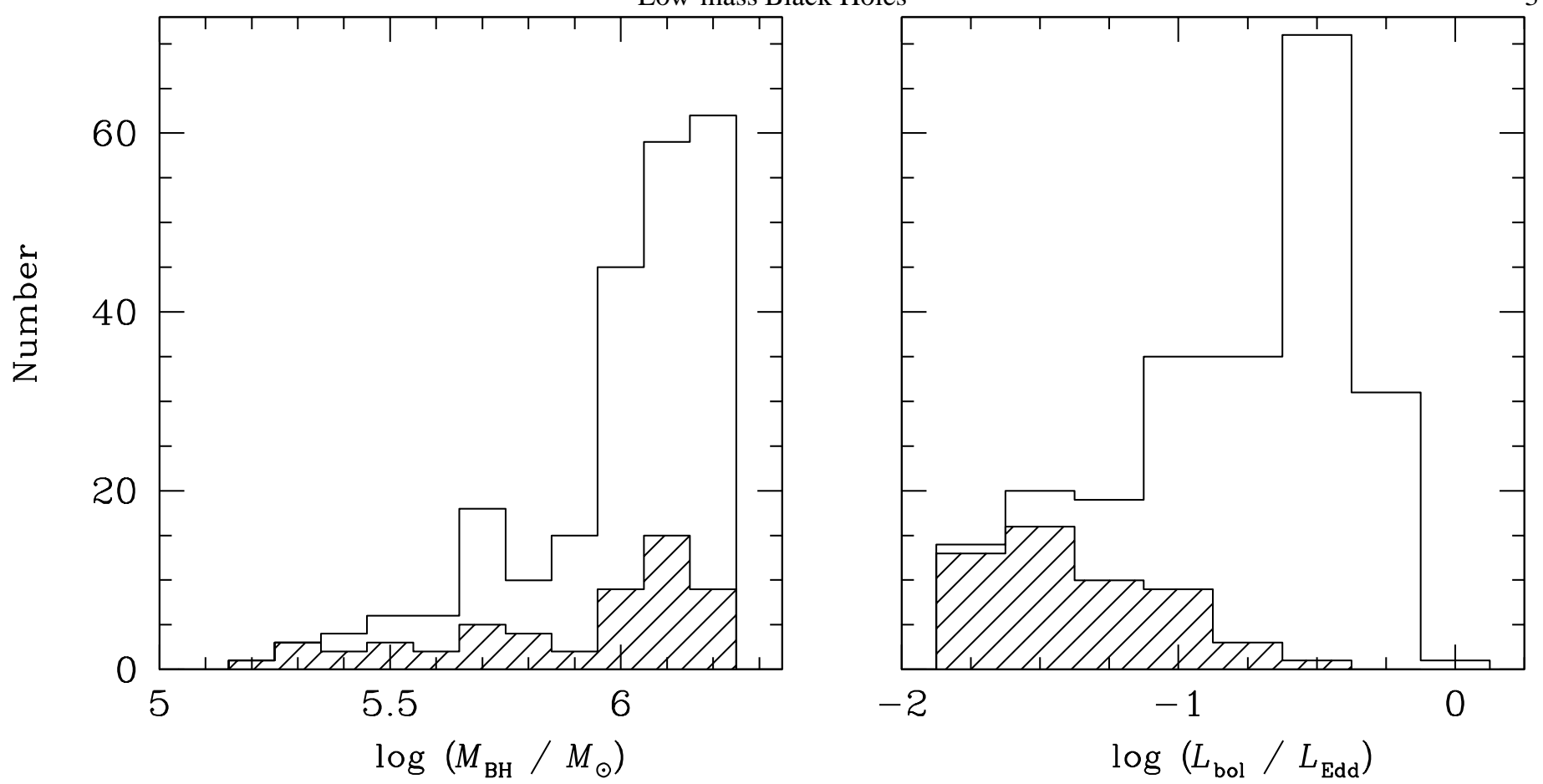

FIG. 1.- Distributions of "virial" BH mass and Eddington ratio for the entire sample. $M_{\mathrm{BH}}$ is calculated from $L_{\mathrm{H} \alpha}$ and FWHM $\mathrm{H}$, using the formalism of Greene

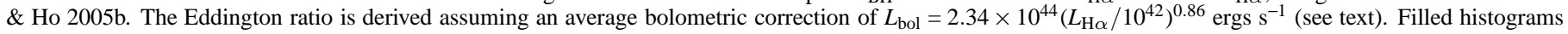
represent objects below the detection threshold defined in Greene \& Ho 2007b (the $c$ sample).

follow standard procedure and model the Fe II emission with an empirical template (e.g., Boroson \& Green 1992; Greene \& Ho 2005b). Since many of our sources also require substantial galaxy subtraction, the Fe II, $\mathrm{H} \beta$, and [O III] lines are modeled simultaneously in the galaxy-continuum-subtracted spectrum, as described below.

In addition to an Fe II template, our model includes narrow [O III], and narrow and broad $\mathrm{H} \beta$. In the standard way, all relative wavelengths for central narrow components are fixed to laboratory values, and the narrow $\mathrm{H} \beta$ flux is fixed to be no more than $1 / 3.1$ of the narrow component of $\mathrm{H} \alpha$ (Case $\mathrm{B}^{\prime}$ recombination; Halpern \& Steiner 1983; Gaskell \& Ferland 1984). The [O III] lines are fit simultaneously with a core and a wing component, following Greene \& Ho (2005a), with a relative strength of 2.96, while the narrow $\mathrm{H} \beta$ line is modeled with a single Gaussian due to its typical low signal-to-noise ratio.

Usually, Fe II templates are derived from $1 \mathrm{Zw} \mathrm{I}$, a strong Fe II source with relatively narrow broad lines. With a broadline width of FWHM $=1240 \mathrm{~km} \mathrm{~s}^{-1}$ (Boroson \& Green 1992), $1 \mathrm{ZW}$ I is significantly broader than many of the objects in our sample. For this reason, we are compelled to build our own Fe II template from the SDSS data. Using a high S/N spectrum of SDSS J155909.62+350147.4, whose FWHM $\mathrm{H}_{\alpha}$ is $860 \mathrm{~km} \mathrm{~s}^{-1}$, we model $\mathrm{H} \beta+[\mathrm{O}$ III $]$ using the 4750-5100 $\AA$ region with no Fe II template included (Fig. 2a), and subtract this model. We further mask the regions 4840-4870 $\AA$ and 5010-5018 $\AA$ (note these wavelengths are in vacuum), since these regions are dominated by noise in the subtraction. While the resulting template is similar to $1 \mathrm{Zw} \mathrm{I}$ (Fig. $2 b$ ), it is clear that we do not recover the shape of the $5000 \AA$ feature perfectly, and that there are slight differences in continuum slope between the two templates. To explore the importance of the former problem, we have repeated our fitting procedure using templates made with various masking regions, and find no difference in the derived fit parameters. The continuum shape, on the other hand, remains a systematic uncertainty inherent to our method; there is some degeneracy between the shape of the continuum and the amplitude of Fe II contamination, leading to uncertainties in the overall amplitude of Fe II. Nevertheless, our measured $\mathrm{H} \beta+[\mathrm{O} \mathrm{III}]$ fits are robust to this uncertainty. When we fix the Fe II template to values ranging from one-half to twice the bestfit value, we find $<1 \%$ changes in all other measured parameters. Presumably the [O III] is sufficiently resolved from the Fe II component at $5000 \AA$ so that small errors in the latter do not strongly impact our model of the former.

\subsection{Comparison with Greene \& Ho (2004)}

Let us examine how the original GH sample compares with this new larger sample. Objects from the original paper will be referenced using their identification number in that paper (e.g., GH02). There are no significant differences between the two works, but there are some issues worth noting. In general, we have improved our selection procedure (see Greene \& Ho 2007b), so that our sample from DR1 is close to two and a half times larger than the original sample. We are now using the $\mathrm{H} \alpha$ rather than the $L_{5100}$ luminosity to derive the BLR radius, and we are using the updated radius-luminosity relation slope of Bentz et al. (2006). Only one (GH19) of the GH objects is not included in the final DR4 broad-line AGN sample of Greene \& Ho (2007b), although GH07, GH11, GH15, GH16, and GH18 now have masses above our mass boundary of $2 \times 10^{6} \mathrm{M}_{\odot}$. Finally, the original GH sample excluded galaxies with significant galaxy contamination, due to the fear that these objects would comprise more massive BHs with low-contrast broad lines that were rendered undetectable by the large galaxy luminosity. In this work we have not explicitly included such a cut, but our EW threshold is operationally similar. While some of the objects in the $c$ sample may consist of high-mass interlopers, 


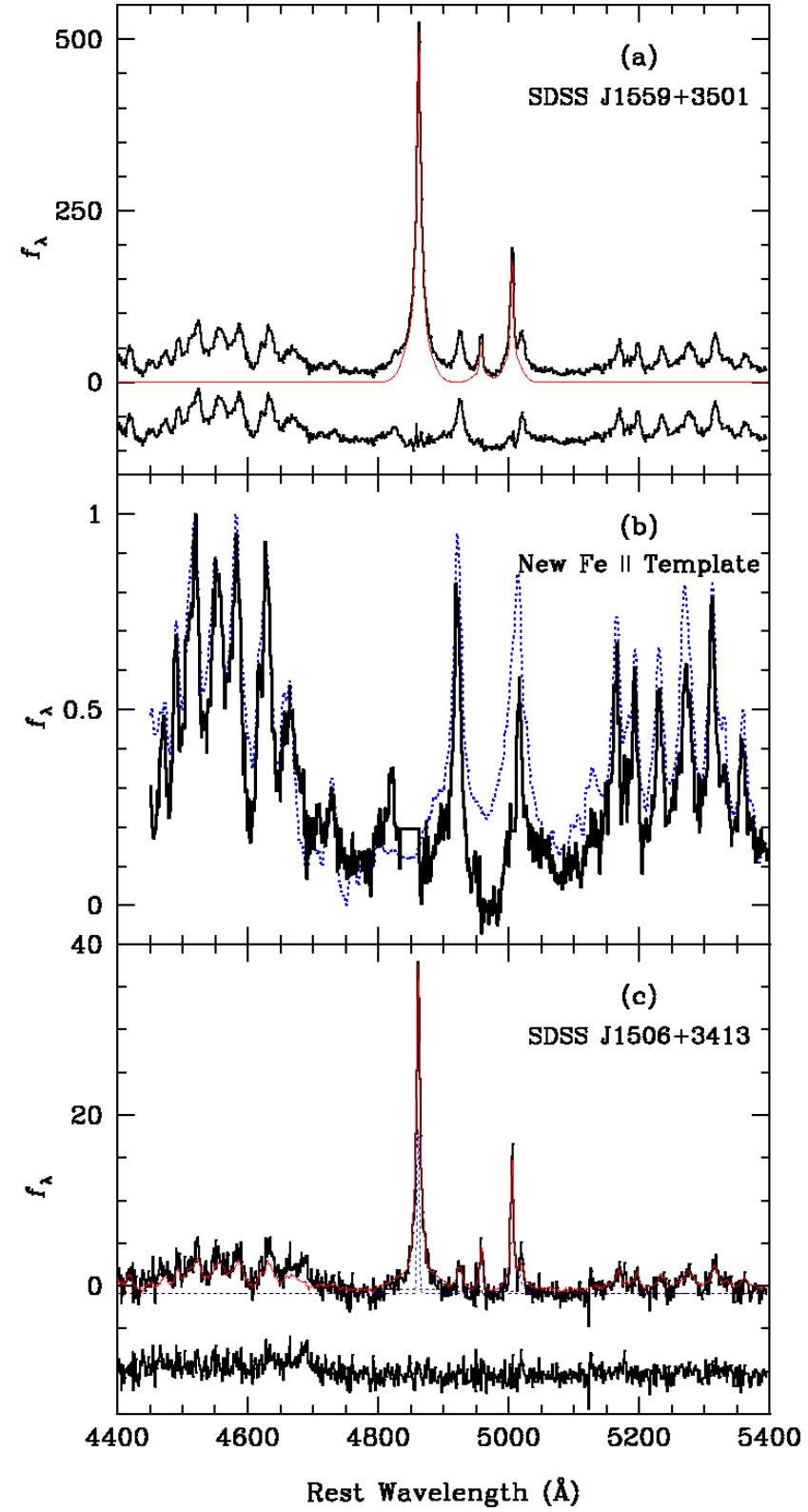

FIG. 2.- (a) The $\mathrm{H} \beta+[\mathrm{O}$ III] fit to SDSS J155909.62+350147 used to create the $\mathrm{Fe}$ II template shown in (b). Shown are the continuum-subtracted (top), best-fit (red thin) and residual (bottom) spectra. Units are $10^{-17} \mathrm{ergs} \mathrm{s}^{-1} \mathrm{~cm}^{-2}$ $\AA^{-1}$. (b) A comparison of the standard Boroson \& Green $19921 \mathrm{ZW}_{\text {I Fe II }}$ template (blue dotted) with that used in this work (solid). Spectra have been normalized to unity. The two templates are very similar, although our template is clearly narrower, and, as discussed in the text, the overall slope is somewhat different. (c) A sample fit to the $\mathrm{H} \beta+[\mathrm{O}$ III] region. Spectra in solid are the continuum-subtracted (top) and residual (bottom) spectra. The total model is overplotted (red thin), along with individual components (blue dotted). Units the same as $(a)$ above.

we find in general very good agreement in properties between the two samples.

\subsection{Comparison with Dong et al. $(2007 a, b)$}

Dong et al. (2007a) have presented a low-mass BH with a mass of $7 \times 10^{4} M_{\odot}$. This source was selected from the Fifth Data Release of the SDSS, and so cannot be cross-checked with our sample. However, the object has been serendipitously observed with $H S T$ and XMM-Newton, as well as being detected with the Röentgen Satellite (ROSAT). There is tentative evidence for variability in the $\mathrm{X}$-ray source $\left(L_{X}=7 \times 10^{40} \mathrm{ergs} \mathrm{s}^{-1}\right)$ and the host galaxy is a disk galaxy with $M_{R}=-17.8 \mathrm{mag}$ and a nuclear bar. The same group has identified a sample of 245 lowmass BHs from DR4, with a mass range of $5 \times 10^{4}-10^{6} M_{\odot}$, and Eddington ratios ranging from 0.02 to 8, comparable to our sample (Dong et al. 2007b). Samples selected from two independent methods will provide important tests on the limitations of our method. Note, however, that the published masses are based on the radius-luminosity relation slope of Kaspi et al. (2005), and would increase by $\sim 0.3$ dex according to the formalism adopted in this paper.

\section{SAMPLE PROPERTIES}

The final sample has a median redshift of 0.086 (0.099 if the $c$ sample is excluded). The original sample has a slightly lower median $z=0.08$ due in part to subsequent deep observations in the Southern Strip (targeting quasars to a limiting magnitude of $i=19.9$ mag rather than $i=19.1 \mathrm{mag}$ ). In Figure 1 we show the distribution of "virial" $\mathrm{BH}$ mass and Eddington ratio for the sample, where $L_{\mathrm{Edd}} \equiv 1.26 \times 10^{38}\left(M_{\mathrm{BH}} / M_{\odot}\right)$ ergs s${ }^{-1}$. Assuming that $L_{\mathrm{bol}}=9.8 L_{5100}$ (McLure \& Dunlop 2004), in terms of $L_{\mathrm{H} \alpha}$ our bolometric correction is $L_{\mathrm{bol}}=$ $2.34 \times 10^{44}\left(L_{\mathrm{H} \alpha} / 10^{42}\right)^{0.86} \mathrm{ergs} \mathrm{s}^{-1}$ (Greene \& Ho 2005b). The median BH mass is $\left\langle M_{\mathrm{BH}}\right\rangle=1.3 \times 10^{6} M_{\odot}$. With a median $\left\langle L_{\text {bol }} / L_{\text {Edd }}\right\rangle=0.4$, we are clearly dominated by sources radiating at substantial fractions of their Eddington limits (Fig. 1). This is not surprising; as discussed by Greene \& Ho (2007b), we are only sensitive to the most luminous BHs in this mass regime.

\subsection{Optical Spectral Properties}

The statistical power of our new enlarged sample provides new constraints on the ensemble physical properties of both the radiating $\mathrm{BHs}$ and their host galaxies. We begin by placing the sources on the two-dimensional diagnostic diagrams that in combination discriminate between a stellar ionizing spectrum or one considerably harder (e.g., Baldwin et al. 1981; Veilleux $\&$ Osterbrock 1987). These line ratios are typically used to divide emission-line galaxies into $\mathrm{H}$ II galaxies and narrow-line AGNs (e.g., Ho et al. 1997a; Kauffmann et al. 2003; Hao et al. 2005; Table 2). In contrast, broad-line-selected objects are unbiased with regard to position on the diagnostic diagrams, while at the same time we know that some fraction of the ionizing continuum is contributed by an AGN.

We focus first on the $[\mathrm{N} \mathrm{II}] \lambda 6583 / \mathrm{H} \alpha$ diagnostic diagram (Fig. 3a). We do not see many objects in the lower-right quadrant. Objects there are low-ionization nuclear emission-line region (LINER; Heckman 1980) sources and typically are highly sub-Eddington AGNs (Ho 2004). Compared to classical Seyfert galaxies, which occupy the upper-right region of the diagnostic diagram, we see that our sources span a broader range in both $[\mathrm{N} \mathrm{II}] / \mathrm{H} \alpha$ and $[\mathrm{O} \mathrm{III}] / \mathrm{H} \beta$. The decrease in $[\mathrm{N} \mathrm{II}] / \mathrm{H} \alpha$ is most easily explained as a decrease in the gas-phase metallicity of these AGNs (e.g., Kraemer et al. 1999; Groves et al. 2006). Since nitrogen is a secondary element, [N II] is particularly sensitive to changes in metallicity for $>0.1 Z_{\odot}$. In contrast, the $[\mathrm{O}$ III $] / \mathrm{H} \beta$ ratio changes much more slowly, because as the metallicity decreases, the temperature of the NLR increases correspondingly, which tends to boost the [O III] strength (e.g., Groves et al. 2004a, 2006; see metallicity tracks on Fig. 4). Relatively low metallicities for this sample are not unexpected, since local AGN hosts in general are known to be massive, bulgedominated galaxies (e.g., Ho et al. 1997b; Kauffmann et al. 
Table 2. Emission-line Measurements

\begin{tabular}{|c|c|c|c|c|c|c|c|c|c|c|c|c|c|}
\hline $\begin{array}{l}\text { ID } \\
\text { (1) }\end{array}$ & $\begin{array}{c}{[\mathrm{O} \text { II }]} \\
\lambda 3727 \\
(2)\end{array}$ & $\begin{array}{c}\text { Fe II } \\
\lambda 4570 \\
(3)\end{array}$ & $\begin{array}{c}(\mathrm{H} \beta)_{\mathrm{n}} \\
(4)\end{array}$ & $\begin{array}{c}(\mathrm{H} \beta)_{\mathrm{b}} \\
(5)\end{array}$ & $\begin{array}{c}{[\mathrm{O} \text { III] }} \\
\lambda 5007 \\
(6)\end{array}$ & $\begin{array}{c}{[\mathrm{O} \text { I] }} \\
\lambda 6300 \\
(7)\end{array}$ & $\begin{array}{r}\mathrm{H} \alpha_{\mathrm{n}} \\
(8)\end{array}$ & $\begin{array}{r}\mathrm{H} \alpha_{\mathrm{b}} \\
(9)\end{array}$ & $\begin{array}{c}{[\mathrm{N} \text { II }]} \\
\lambda 6583 \\
(10)\end{array}$ & $\begin{array}{c}{[\mathrm{S}} \\
\lambda \mathrm{II}] \\
\lambda 6716 \\
(11)\end{array}$ & $\begin{array}{c}{[\mathrm{S} \text { II }]} \\
\lambda 6731 \\
(12)\end{array}$ & $\begin{array}{c}\mathrm{FWHM}_{\mathrm{H} \alpha} \\
\left(\mathrm{km} \mathrm{s}^{-1}\right) \\
(13)\end{array}$ & $\begin{array}{c}\mathrm{FWHM}_{[\mathrm{OIII}]} \\
\left(\mathrm{km} \mathrm{s}^{-1}\right) \\
(14)\end{array}$ \\
\hline 1 & 0.46 & 0.18 & 0.27 & $<0.41$ & 1.84 & 0.067 & 1.4 & 1.3 & 0.51 & 0.31 & 0.20 & 1870 & 230 \\
\hline 2 & 1.2 & 1.6 & 0.57 & 1.0 & 0.971 & 0.16 & 1.9 & 4.8 & 0.79 & 0.43 & 0.33 & 976 & 162 \\
\hline 3 & 0.53 & $<0.39$ & 0.18 & 0.62 & 1.88 & 0.10 & 0.69 & 0.84 & 0.58 & 0.24 & 0.20 & 1710 & 215 \\
\hline
\end{tabular}

\footnotetext{
Note. - Col. (1): Identification number assigned in this paper. Col. (2)-(12): All fluxes are relative to that of [O III] $\lambda 5007$, which is listed in units of $10^{-17} \mathrm{ergs} \mathrm{s}^{-1} \mathrm{~cm}^{-2}$. Note that these are observed values; only Galactic extinction correction has been applied. The subscripts " $n$ " and "b" in Col. (4)-(5) and (8)-(9) refer to the narrow and broad components of the line, respectively. Col. (13)-(14): Linewidths have been corrected for instrumental resolution using the values measured from arc spectra and tabulated by the SDSS. Table 2 is available in its entirety via the link to the machine-readable table above. The following is included only as a guide to content and presentation.
}

2003), while these low-mass BHs tend to live in low-mass and correspondingly low-metallicity systems $(\$ 3.4$; Tremonti et al. 2004).

More striking is the apparent corresponding decrease in $[\mathrm{O} \mathrm{III}] / \mathrm{H} \beta$, which as we have seen, cannot be ascribed to a decrease in metallicity. In fact, $[\mathrm{O}$ III $] / \mathrm{H} \beta$ appears to decrease in concert with $[\mathrm{N} \mathrm{II}] / \mathrm{H} \alpha$, [S II] $/ \mathrm{H} \alpha$, and [O I] $\lambda 6300 / \mathrm{H} \alpha$ (Fig. $3 b, c)$. At the same time, there is a clear trend of increasing [O II $] \lambda 3727 /[\mathrm{O}$ III $]$ line ratios as the $[\mathrm{O} \mathrm{III}] / \mathrm{H} \beta$ ratio decreases (Fig. 4). Changes in a variety of NLR conditions, including metallicity, electron density $\left(n_{e}\right)$, ionization parameter $(U)$, ionizing spectral shape, and reddening might be invoked to explain the observed line ratios, while particular properties of either the AGN (e.g., luminosity or Eddington ratio) or the host galaxy (e.g., star formation) may be responsible for the changing conditions. As we will argue, it is unlikely that a single parameter is responsible for all of these trends. Correlated errors might also be expected to move line ratios in particular ways across the diagnostic diagrams. If, for instance, we are systematically overestimating the strength of narrow $\mathrm{H} \alpha$ in systems with weak NLR emission, then we might expect correlated tracks as observed. However, [O III] is never blended with broad $\mathrm{H} \beta$ in these sources, and thus [O III] errors are not correlated with the other lines, but nevertheless the [O III] line strength spans a wide range relative to $\mathrm{H} \beta$.

One might imagine that the intrinsic line ratios are all located in the Seyfert locus, but that contamination from star formation spreads out their positions in the diagnostic diagrams. We perform a simple thought experiment to demonstrate that there must be an intrinsic spread in line ratios to reproduce the observed trends. Since star-forming galaxies follow a welldefined sequence in the diagnostic diagram (bounded by the Kauffmann et al. 2003 relation shown Fig. 5), we can easily investigate the type of bias that star formation would contribute. In Figure 5 we have chosen three fiducial AGN positions in the diagnostic diagram. Position A represents a typical high-ionization system, while $\mathrm{B}$ has low $[\mathrm{O} \mathrm{III}] / \mathrm{H} \beta$ and $[\mathrm{N} \mathrm{II}] / \mathrm{H} \alpha$ and $\mathrm{C}$ represents a LINER. To each AGN we add varying amounts of star formation with line ratios drawn from the Kauffmann line, and the $\mathrm{H} \beta$ strength in star formation varying from half to twice the AGN $\mathrm{H} \beta$ luminosity. The tracks (shown as dash-dot, solid, and dashed lines for positions A, $\mathrm{B}$, and $\mathrm{C}$, respectively) demonstrate that while star formation may increase the spread in positions on the diagnostic diagram, it alone cannot move objects from the high-ionization position A to the low-[O III $] / \mathrm{H} \beta$ positions we observe. There must be a true spread in the intrinsic AGN line ratios. Furthermore, position $\mathrm{C}$ is disfavored, since only with a large fraction of the light contributed by low-metallicity star formation might we move the objects from position $\mathrm{C}$ toward position $\mathrm{B}$. If the objects in this sample follow the mass-metallicity relation of Tremonti et al. (2004), with typical luminosities of $M_{g} \approx-19.3$ (Table 3, $\S 3.4$ ), they would not, on average, have sub-solar metallicity.

Given that the dispersion in line ratios must be intrinsic to the NLR, we investigate various means to explain the observed line-ratio distributions. Ho et al. (1993) find that narrow-line AGNs display a similar trend in the occupation of diagnostic diagrams, which they ascribe to a trend of harder ionizing spectral shape at lower luminosity. Exactly this trend has been seen in the X-ray to optical slopes of broad-line AGNs over a wide range in luminosity (e.g., Strateva et al. 2005), including for a subset of the GH sample (Greene \& Ho 2007a). A correlation between low-luminosities and harder spectral shape would lead to a correlation between AGN luminosity and line ratios, but we see no trends between AGN luminosity (as traced by $L_{\mathrm{H} \alpha}$ ) and either $[\mathrm{O} \mathrm{III}] / \mathrm{H} \beta$ or $[\mathrm{O} \mathrm{I}] / \mathrm{H} \alpha$.

We have already discussed the impact of varying metallicity above, and since $[\mathrm{N} \mathrm{II}] / \mathrm{H} \alpha$ is most sensitive to its variation, metallicity is unlikely to explain the correlated decrease in $[\mathrm{S} \mathrm{II}] / \mathrm{H} \alpha,[\mathrm{O} \mathrm{I}] / \mathrm{H} \alpha$ and $[\mathrm{N} \mathrm{II}] / \mathrm{H} \alpha$. Furthermore, metallicity variations alone cannot explain the large observed range in $[\mathrm{O} \mathrm{II}] /[\mathrm{O} \mathrm{III}]$ ratios (Fig. 4). Changes in either ionization parameter or density might explain the $[\mathrm{O}$ II $] /[\mathrm{O}$ III] ratios; increases in either leads to a relative increase in [O III]. Indeed, electron densities inferred from the ratio of the [S II] lines show a significant correlation with $[\mathrm{O}$ III $] / \mathrm{H} \beta$. The Spearman rank correlation coefficient is $\rho=0.296$, with a probability $P<10^{-4}$ that no correlation is present. However, there is only a mildly significant trend between electron density and $[\mathrm{N}$ II $] / \mathrm{H} \alpha(\rho=0.197, P=0.003)$ and no trend with $[\mathrm{S} \mathrm{II}] / \mathrm{H} \alpha$ ( $\rho=0.124, P=0.06$; as one might expect given their lower critical densities). On the other hand, changes in ionization parameter move, for example, $[\mathrm{N} \mathrm{II}] / \mathrm{H} \alpha$ vs. $[\mathrm{O}$ III $] / \mathrm{H} \beta$ on tracks perpendicular to those observed (e.g., Groves et al. 2004a, b). A single parameter seems unable to reproduce all of the observed trends simultaneously. Presumably this is due, in part, to stratification in the density and ionization parameter of the NLR with radius, which leads to different typical conditions in the emission regions of different lines (e.g., Filippenko \& Halpern 1984).

In summary, a single parameter cannot be invoked to explain the observed positions in the diagnostic diagrams. 


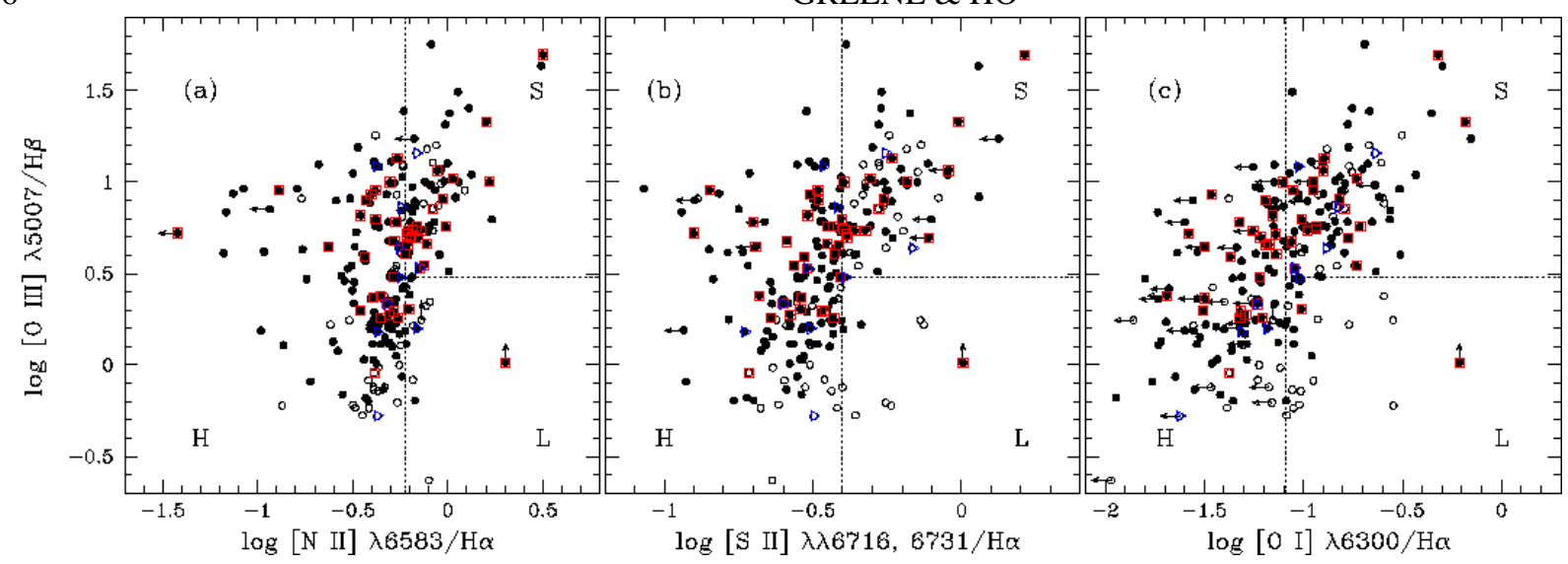

FIG. 3.- Diagnostic diagrams plotting $\log [\mathrm{O}$ III $] \lambda 5007 / \mathrm{H} \beta$ versus $(a) \log \left[\mathrm{N}\right.$ II] $\lambda 6583 / \mathrm{H} \alpha,(b) \log \left[\mathrm{S}_{\text {II }}\right] \lambda \lambda 6716,6731 / \mathrm{H} \alpha$, and $(c) \log [\mathrm{O}$ I] $\lambda 6300 / \mathrm{H} \alpha$. The $c$ sample is shown in open symbols, and there are red boxes around ROSAT detections and blue triangles around FIRST detections. The line ratios have not been corrected for reddening, but this should not matter because of the small wavelength separation of the lines. The dotted lines mark the boundaries of the three main classes of emission-line nuclei, according to the convention of Ho et al. 1997a: "H" = H n nuclei, "S" = Seyferts, and "L" = LINERs.

Table 3. Luminosity and Mass Measurements

\begin{tabular}{cccccccc}
\hline \hline $\begin{array}{c}\text { ID } \\
(1)\end{array}$ & $\begin{array}{c}M_{g} \text { (total) } \\
(2)\end{array}$ & $\begin{array}{c}M_{g}(\mathrm{AGN}) \\
(3)\end{array}$ & $\begin{array}{c}M_{g} \text { (host) } \\
(4)\end{array}$ & $\begin{array}{c}\log L_{\mathrm{H} \alpha} \\
(5)\end{array}$ & $\begin{array}{c}\log M_{\mathrm{BH}} \\
(6)\end{array}$ & $\begin{array}{c}\log L_{\mathrm{bol}} / L_{\mathrm{Edd}} \\
(7)\end{array}$ & $\begin{array}{c}\log M_{\mathrm{OLD}} \\
(8)\end{array}$ \\
\hline 1 & -18.02 & -15.91 & -17.82 & 40.11 & 6.2 & -1.6 & 5.8 \\
2 & -20.16 & -18.24 & -19.91 & 41.22 & 6.1 & -0.5 & 5.9 \\
3 & -19.44 & -16.31 & -19.37 & 40.26 & 6.2 & -1.4 & $\cdots$ \\
\hline
\end{tabular}

Note. - Col. (1): Identification number assigned in this paper. Col. (2): Total $g$-band absolute magnitude. Col. (3): AGN $g$-band absolute magnitude, estimated from $L_{\mathrm{H} \alpha}$ given in Col. (5) and a conversion from $L_{\mathrm{H} \alpha}$ to $M_{g}$ assuming $f_{\lambda} \propto \lambda^{-1.56}$. Col. (4): Host galaxy $g$-band absolute magnitude, obtained by subtracting the AGN luminosity from the total luminosity. Cases where the AGN accounts for the total galaxy luminosity are indicated with an ellipsis. Col. (5): AGN luminosity in broad $\mathrm{H} \alpha$ (ergs s$\left.{ }^{-1}\right)$. Col. (6): Virial mass estimate of the $\mathrm{BH}\left(M_{\odot}\right)$. Col. (7): Ratio of the bolometric luminosity (see text) to the Eddington luminosity. Col. (8): Previously reported virial mass estimate of the $\mathrm{BH}\left(M_{\odot}\right)$ from Greene \& $\mathrm{Ho}_{0} 2007 \mathrm{~b}$. There are no entries for the $c$ sample. Table 3 is available in its entirety via the link to the machine-readable table above. The following is included only as a guide to content and presentation.

Although changing physical conditions (such as electron density) within the NLR may be important, the objects lying close to the star-forming locus may simply have an extremely weak NLR, such that the majority of the narrow emission is coming from $\mathrm{H}$ II regions. A similar phenomenon, the "vanishing" NLR, has been seen in a substantial fraction of higher redshift AGNs at high Eddington ratios (e.g., Netzer et al. 2004, 2006). If observed correlations between NLR size and AGN luminosity (e.g., Bennert et al. 2002; Schmitt et al. 2003) are extrapolated to the highest luminosities, then one would predict unbound nebulosities; Netzer et al. (2004) suppose that the NLRs in these systems grew so large that they were no longer bound to their host galaxies. The luminosities in our systems are significantly lower, and the expected NLR sizes are $<100 \mathrm{pc}$ if they obey the NLR size-luminosity relation, but we cannot rule out that the AGN actually expelled some large fraction of the surrounding ISM, leading to overall weakness in the line intensities.

Finally, there is an apparent connection between $[\mathrm{O}$ III $] / \mathrm{H} \beta$ and Eddington ratio (e.g., Boroson 2002). However, for these objects there is no trend between $[\mathrm{O} \mathrm{III]} / \mathrm{H} \beta$ (narrow) and $L_{\text {bol }} / L_{\text {Edd }}(\rho=0.043, P=0.5)$ or [O III] equivalent width and $L_{\text {bol }} / L_{\text {Edd }}(\rho=0.093, P=0.2)$. These trends do not change when the $c$ sample is excluded. On the other hand, although no physically motivated explanation exists, low $[\mathrm{O}$ III $] / \mathrm{H} \beta$ traditionally was a defining characteristic of the class of objects known as narrow-line Seyfert 1 galaxies (NLS1s; Osterbrock \& Pogge 1985),
Table 4. ROSAT Detections

\begin{tabular}{ccccc}
\hline \hline ID & $C$ & $\log N_{\mathrm{H}}$ & $\log f_{X}$ & $\log L_{X}$ \\
$(1)$ & $(2)$ & $(3)$ & $(4)$ & $(5)$ \\
\hline 18 & $0.023 \pm 0.011$ & 20.55 & $-12.66 \pm 0.16$ & $42.44 \pm 0.16$ \\
24 & $0.053 \pm 0.019$ & 20.86 & $-12.17 \pm 0.05$ & $42.49 \pm 0.05$ \\
28 & $0.024 \pm 0.0090$ & 20.79 & $-12.54 \pm 0.07$ & $42.19 \pm 0.07$ \\
\hline
\end{tabular}

Note. - Col. (1): Identification number assigned in this paper. Col. (2): ROSAT count rate (counts $\mathrm{sec}^{-1}$ ). Col. (3): Galactic column $N_{\mathrm{H}}\left(\mathrm{cm}^{-2}\right)$, calculated following Dickey \& Lockman 1990. Col. (4): X-ray flux in the $0.5-2 \mathrm{keV}$ band $\left(\mathrm{ergs} \mathrm{s}^{-1}\right.$ $\mathrm{cm}^{-2}$ ) assuming a power-law spectrum with $\Gamma_{s}=2$ and $N_{\mathrm{H}}$ from col. (3). Col. (5): X-ray luminosity in the $0.5-2 \mathrm{keV}$ band $\left(\operatorname{ergs~s}{ }^{-1}\right)$. Table 4 is available in its entirety via the link to the machine-readable table above. The following is included only as a guide to content and presentation.

which are broad-line AGNs with relatively narrow broad-line widths. We turn now to a general comparison of our objects with NLS1s.

\subsection{Comparison with NLS1s}

While NLS1s are an observationally defined class, a picture has emerged, based on their strong $\mathrm{Fe} \mathrm{II/H} \beta$ and weak $[\mathrm{O}$ III $] / \mathrm{H} \beta$ line ratios, strong soft X-ray excess (e.g., Boller et al. 1996) and X-ray variability (Leighly 1999), that NLS1s are in general low-mass BHs (thus accounting for the relatively narrow broad lines) in a high accretion state (e.g., Pounds et al. 1995). Since the current sample is selected based on BH mass (and indirectly $L_{\text {bol }} / L_{\text {Edd }}$ ), it represents a very uniform, optically selected NLS1 sample. In this section, we highlight comparisons between the optical and broad spectral properties of this and other NLS1 samples.

One of the supporting pieces of evidence that NLS1s are radiating close to their Eddington limit comes from their observed high $\mathrm{Fe} \mathrm{II} / \mathrm{H} \beta$ ratios (e.g., Véron-Cetty et al. 2001; throughout this section we refer to total [broad+narrow] $\mathrm{H} \beta$ luminosity). Using principle component analysis, Boroson \& Green (1992) found that NLS1s lie at one extreme of their first component (fondly known as Eigenvector 1), and later work has shown that Eigenvector 1 properties, including high $\mathrm{Fe} \mathrm{II} / \mathrm{H} \beta$ and low $[\mathrm{O} \mathrm{III}] / \mathrm{H} \beta$ ratios, 


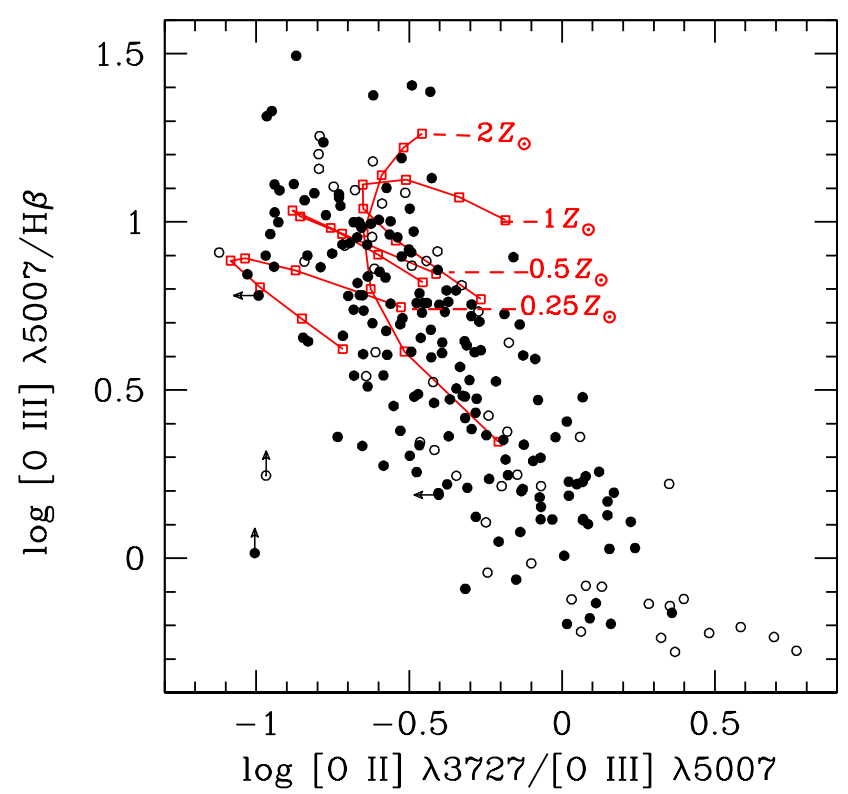

FIG. 4.- Tracks in $\left[\begin{array}{ll}\mathrm{O} & \mathrm{II}\end{array}\right][\mathrm{O} \mathrm{III}]$ for different metallicities, following Groves et al. 2006. At a given metallicity, the tracks span a range in ionization parameter $\tilde{\mathrm{U}}_{0} \equiv S_{*} /\left(c \tilde{\mathrm{n}}_{\mathrm{H}}\right)$, where $S_{*}$ is the number of ionizing photons, and $\tilde{\mathrm{n}}_{\mathrm{H}}=P_{\mathrm{gas}} /\left(k 10^{4}\right)$. The model values are log $\tilde{\mathrm{U}}_{0}=(-0.40,-1.03,-1.43,-1.88,-2.24,-2.57,-2.98)$ and the tracks are labeled at the low- $\tilde{U}_{0}$ end, as indicated with dashed lines. It is clear that metallicity alone cannot account for the spread in $\left[\mathrm{O}_{\mathrm{II}}\right] /[\mathrm{O} \mathrm{II}]$ ratios we observe. Open symbols indicate the $c$ sample.

as well as soft X-ray excess and radio weakness, tend to be correlated with Eddington ratio (e.g., Boroson 2002). On the other hand, GH found that their objects span a larger range in both Fe II and [O III] strengths relative to $\mathrm{H} \beta$ than classic NLS1s.

The current sample has somewhat lower $\mathrm{Fe} \mathrm{II} / \mathrm{H} \beta$ strengths than GH found, perhaps because there are objects with more significant galaxy contamination in this sample. Using the Kaplan-Meier product-limit estimator, which accounts for upper limits (Feigelson \& Nelson 1985), we find $\langle\mathrm{Fe} \mathrm{II} / \mathrm{H} \beta\rangle=$ $0.57 \pm 0.03(0.61 \pm 0.03)$ with (without) the $c$ sample, as compared to $\langle\mathrm{Fe} \mathrm{II} / \mathrm{H} \beta\rangle=0.67 \pm 0.04$ for the $56 \mathrm{NLS} 1 \mathrm{~s}$ presented by Véron-Cetty et al. (2001), and $\langle\mathrm{Fe} \mathrm{II} / \mathrm{H} \beta\rangle=1.32 \pm 0.16$ for the original GH sample. The [O III] distributions are consistent with our previous findings: $\langle[\mathrm{O} \mathrm{III}] / \mathrm{H} \beta\rangle=2.6 \pm 0.3(1.9 \pm 0.2)$, while we found $\langle[\mathrm{O} \mathrm{III}] / \mathrm{H} \beta\rangle=2.24 \pm 0.72$ for the original sample. Although it has been customary to cite $[\mathrm{O} \mathrm{III}] / \mathrm{H} \beta$ (total), the ratio of $[\mathrm{O} \mathrm{III]}] / \mathrm{H} \beta$ (narrow) is more straightforward to interpret, and we report it as well: $\langle[\mathrm{O} \mathrm{III}] / \mathrm{H} \beta$ (narrow) $\rangle=6.1 \pm 0.5$ $(6.5 \pm 0.6)$. In all other discussion, we report trends for $[\mathrm{O} \mathrm{III}] / \mathrm{H} \beta$ (narrow).

More generally, trends in spectral properties with Eddington ratio may be manifest in composite spectra. We investigate four bins of increasing Eddington ratio (similar to increasing bins in luminosity over such a restricted range in $\mathrm{BH}$ mass; Fig. 6), and we have included the $c$ objects, which comprise the lowest $L_{\mathrm{bol}} / L_{\text {Edd }}$ bin. To compute the composite, each spectrum is normalized to the median value in the spectral regions $4100-4800$ $\AA$ and 5100-5800 $\AA$, resampled onto the same wavelength grid and then median combined. Median stacking preserves relative line ratios, but not the continuum shape (e.g., Francis et al. 1991). Although we do not explicitly weight the spectra, they are each normalized to their respective continuum level, which gives extra weight to faint sources. Errors at each pixel are calculated as the $68 \%$ interquartile range of the included pixel

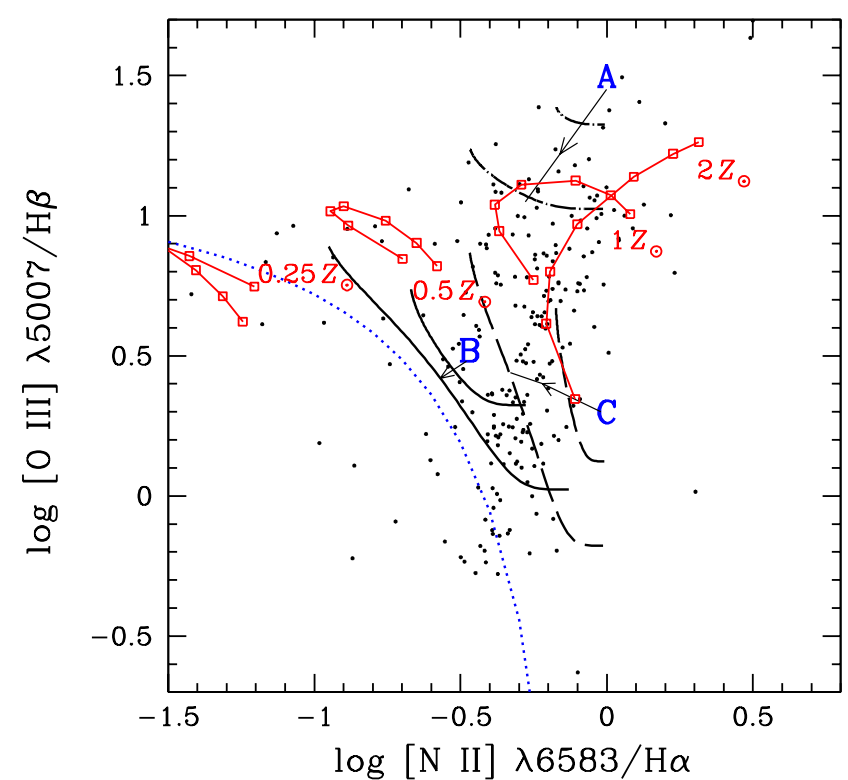

FIG. 5.- A diagnostic diagram highlighting how different effects move points in the parameter space. For pure AGNs with line ratios originating at positions $\mathrm{A}, \mathrm{B}$, and $\mathrm{C}$, we have mixed star-forming galaxies with line ratios along the Kauffmann et al. 2003 line (blue dotted), assuming that the $\mathrm{H} \beta$ strength from photoionization by starlight ranges from half (upper track; A in long dash-dot, $\mathrm{B}$ in solid, $\mathrm{C}$ in long dash) to twice (lower track) that by the AGN. The thin arrows indicate the trajectory of increasing contributions from star formation. Also shown are photoionization models from Groves et al. 2006. Each track covers a single metallicity and the same range of ionization parameters as Figure 4 above. While mixing stellar and AGN-excited line ratios may broaden the locus of points in the diagnostic diagrams, it is clear that the intrinsic line ratios do not all originate at position $\mathrm{A}$.

values, following, e.g., Fine et al. (2006). Note that this procedure is considerably simpler than that of, e.g., Francis et al. (1991), since the redshifts measured by SDSS are highly reliable and the spectral coverage and depth are so uniform for the majority of the sample. The clearest trend as we move to higher Eddington ratio is the decreasing importance of the galaxy continuum. At the same time, the Fe II features are much more apparent in the higher Eddington ratio bins. This appears to be in keeping with our expectations from Eigenvector 1. On the other hand, using the original, unbinned data, we do not find significant evidence for a correlation between Fe II $/ \mathrm{H} \beta$ and $L_{\text {bol }} / L_{\text {Edd }}(\rho=-0.07, P=0.3)$. There is a decrease in the $[\mathrm{O} \mathrm{III}] / \mathrm{H} \beta$ (total) ratio with Eddington ratio in the composite spectra, which ranges from $3.6 \pm 1.6$ in the lowest $L_{\text {bol }} / L_{\text {Edd }}$ bin to $0.65 \pm 0.03$ (or $2.4 \pm 0.3$ when considering only the narrow $\mathrm{H} \beta$ ). Errors are dominated by the variance in line strengths for individual objects comprising the composite.

\subsection{Radio and X-ray Spectral Properties}

There is additional information about the accretion properties of the sample in their broader spectral energy distributions. NLS1s, in general, are known for their strong soft X-ray excess, but Greene \& Ho (2007a; see also Williams et al. 2004) have found that objects selected purely on the basis of mass are not as extreme in this regard as classic NLS1s. In this case, using $5 \mathrm{ks}$ observations of the 10 nearest GH objects, they found that the objects had very typical $0.5-2 \mathrm{keV}$ power-law slopes of $\Gamma_{s} \approx 1.8$ [where $N(E) \propto E^{-\Gamma_{s}}$ ]. Within the current sample, 55 are detected by the ROSAT All-Sky Survey (Voges et al. 1999), of which only two are in the $c$ sample, with fluxes ranging from $5.9 \times 10^{-14}$ to $3.4 \times 10^{-12} \mathrm{ergs} \mathrm{s}^{-1} \mathrm{~cm}^{-2}$. Fluxes are derived from the ROSAT count rates using WebP imms (Mukai 1993) 


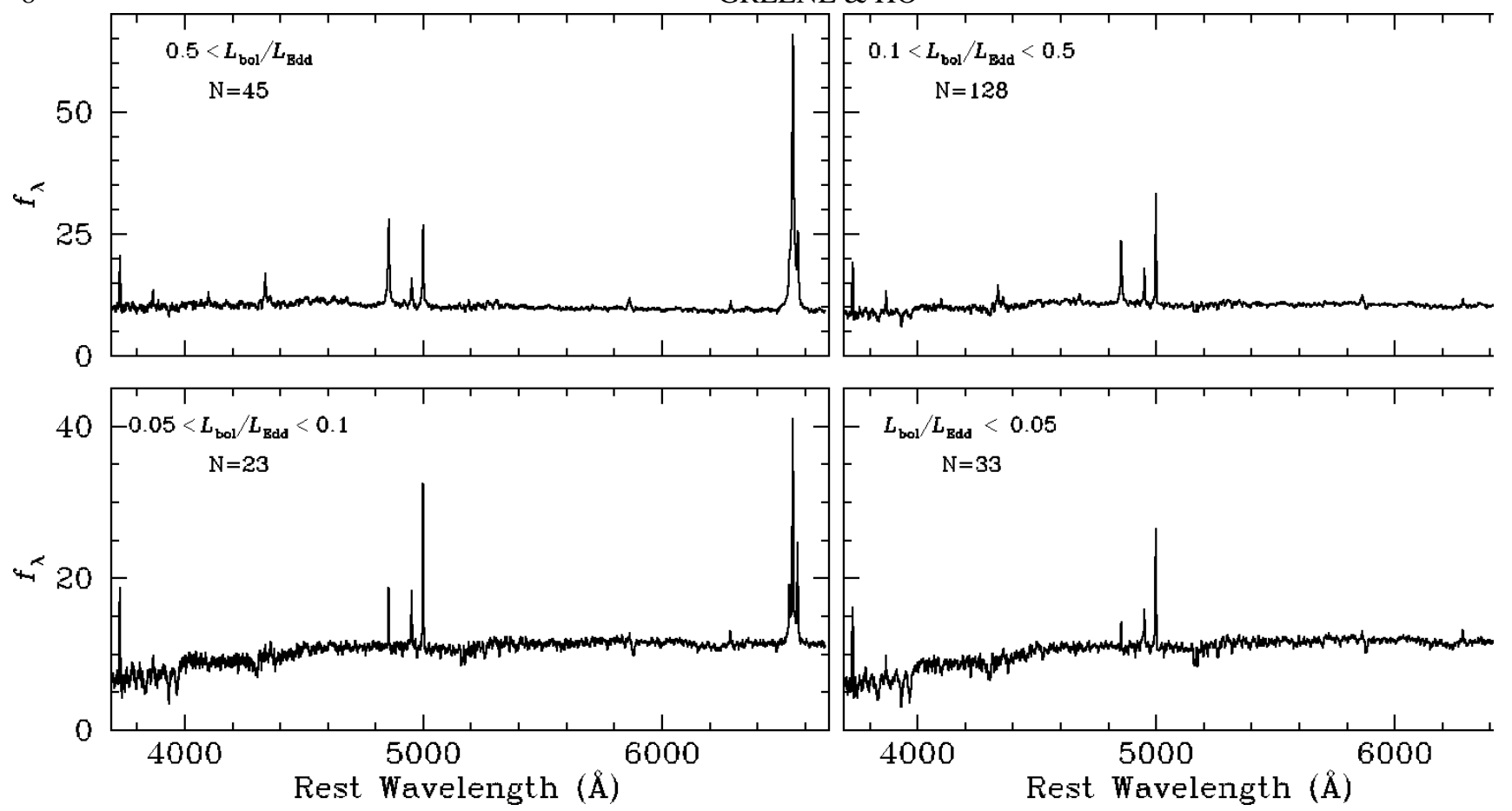

FIG. 6. - Composite spectra in bins of increasing Eddington ratio, as shown. Each spectrum is the median combination of all spectra in the indicated $L_{\mathrm{bol}} / L_{\mathrm{Edd}}$ bin, with the total number of galaxies indicated. Each spectrum is normalized to emission-line-free bands prior to combination (see text for details). Note the increasing strength of the $\mathrm{Fe}$ II features and decreasing galaxy continuum strength with increasing Eddington ratio.

and assuming a slope of $\Gamma_{s}=2$. In calculating uncertainties in the fluxes, $\Gamma_{s}$ is allowed to vary from 1 to 3 , which is the full range observed by Greene \& Ho (2007a). The corresponding range of $0.5-2 \mathrm{keV}$ luminosities is $10^{40}$ to $7 \times 10^{43} \mathrm{ergs} \mathrm{s}^{-1}$ (Table 4). Since inactive galaxies with optical luminosities similar to those of the galaxies in our sample $\left(L_{B} \approx 10^{9}-10^{10} L_{\odot}\right)$ are expected to have typical X-ray luminosities of $\sim 10^{39} \mathrm{ergs} \mathrm{s}^{-1}$ (e.g., Fabbiano 1989), the AGN most likely dominates the Xray emission from the majority of these sources.

Customarily, the ratio of optical to X-ray luminosity is expressed in terms of the slope of a supposed power-law between the optical and X-ray bands, $\alpha_{\mathrm{ox}} \equiv-0.3838 \log \left(f_{2500} / f_{2 \mathrm{keV}}\right)$. The flux density at $2500 \AA, f_{2500}$, is calculated using the measured $L_{\mathrm{H} \alpha}$, the relation between $L_{\mathrm{H} \alpha}$ and $L_{5100}$ from Greene $\&$ Ho (2005b), and an assumed spectral slope of $\beta=-1.56$ $\left(f_{\lambda} \propto \lambda^{-\beta}\right.$; Vanden Berk et al. 2001). For our sample, the values lie in the range $-1.5<\alpha_{\mathrm{ox}}<-0.69$, with a median of $\left\langle\alpha_{\mathrm{ox}}\right\rangle=-1.04$. We would expect an $\alpha_{\mathrm{ox}} \approx-1.2$ corresponding to the median $l_{2500}=6 \times 10^{27} \mathrm{ergs} \mathrm{s}^{-1} \mathrm{~Hz}^{-1}$ (as was seen for the GH objects observed with Chandra; Greene \& Ho 2007a). While the observed median is slightly higher than one would expect (e.g., Strateva et al. 2005; Steffen et al. 2006), it is within the observed scatter, and given that these are the ROSAT-detected members of our sample, it is not surprising to find that they are X-ray bright. We see no obvious trends between $\alpha_{\text {ox }}$ and $L_{\mathrm{bol}} / L_{\mathrm{Edd}}$ or Fe II $/ \mathrm{H} \beta$, but we caution that the dynamic range of this sample is very limited, and that we do not have meaningful upper limits for the X-ray-undetected sources.

Radio emission is a complementary probe of the accretion process, if difficult to interpret cleanly. In general, NLS1s are radio-quiet (see review in Greene et al. 2006), where radio-quiet typically means $R<10\left(R \equiv f_{6 \mathrm{~cm}} / f_{4400}\right)$. Weak radio emission is part of Eigenvector 1 (e.g., Boroson \& Green 1992), and mirrors the general tendency for $\mathrm{BHs}$ of all masses to be radio-quiet when in high accretion states (e.g., McClintock \& Remillard 2006). Also, Best et al. (2005) find a strong preference for radio sources to live in massive galaxies. A low incidence of radio activity in this sample is thus unsurprising. Eleven of the objects are detected by the VLA ${ }^{3}$ FIRST survey at $20 \mathrm{~cm}$ although all but 18 objects are within the FIRST footprint (Becker et al. 1995. The radio sources at $20 \mathrm{~cm}$ are unresolved in all cases, with radio powers that range from $2 \times 10^{21}$ to $9 \times 10^{22} \mathrm{~W} \mathrm{~Hz}^{-1}$ (Table 5). Inactive star-forming galaxies can easily produce this level of radio emission (e.g., Condon 1989), which need not be AGN-dominated. The exception is GH10, which we have imaged with the VLA in A-configuration at $6 \mathrm{~cm}$ (Greene et al. 2006). We find that the source remains completely unresolved, suggesting that the AGN provides the dominant source of emission from this galaxy.

Of the objects with radio emission, four are in the $c$ sample and five others are radio-quiet, which translates into a radioloud fraction of $1 \%$ (accounting for the 18 objects in the sample that are outside of the FIRST footprint), to be compared with the range of $0 \%-6 \%$ for NLS1s in the literature (Greene et al. 2006). In Figure 7 the detected sources are placed on the relation between radio power and [O III] luminosity observed for local AGNs. They are roughly consistent with the relation measured for Seyfert galaxies (Ho \& Peng 2001), but they are systematically higher than the fiducial relation. In addition, we have retrieved FIRST cut-outs around each non-detection. We use these images to derive upper limits on the radio luminosity (Table 5), and we also stack the images in two bins divided at an [O III] luminosity of $L_{[\mathrm{OIII}]}=3 \times 10^{40} \mathrm{ergs} \mathrm{s}^{-1}$. Because FIRST is a uniform survey, no weighting is applied, but in 16 cases

\footnotetext{
${ }^{3}$ The VLA is operated by the National Radio Astronomy Observatory, which is a facility of the National Science Foundation, operated under cooperative agreement by Associated Universities, Inc
} 
Table 5. FIRST Detections

\begin{tabular}{rccc}
\hline \hline ID & $\begin{array}{c}S_{20 \mathrm{~cm}} \\
(1)\end{array}$ & $\begin{array}{c}\log P_{20 \mathrm{~cm}} \\
(2)\end{array}$ & $\begin{array}{c}\log R \\
(4)\end{array}$ \\
\hline 47 & $1.77 \pm 0.14$ & $21.41 \pm 0.04$ & 0.65 \\
69 & $1.43 \pm 0.15$ & $22.87 \pm 0.05$ & 0.96 \\
87 & $1.03 \pm 0.22$ & $22.19 \pm 0.10$ & 0.60 \\
101 & $2.41 \pm 0.14$ & $22.60 \pm 0.03$ & 1.90 \\
106 & $5.96 \pm 0.17$ & $22.19 \pm 0.01$ & 1.30 \\
140 & $1.03 \pm 0.15$ & $21.94 \pm 0.07$ & 1.20 \\
146 & $1.29 \pm 0.15$ & $22.31 \pm 0.05$ & 0.44 \\
158 & $1.55 \pm 0.15$ & $21.87 \pm 0.04$ & 0.89 \\
163 & $2.33 \pm 0.14$ & $21.37 \pm 0.03$ & 1.10 \\
174 & $1.91 \pm 0.15$ & $22.95 \pm 0.04$ & 1.20 \\
203 & $3.39 \pm 0.14$ & $21.87 \pm 0.02$ & 0.00 \\
\hline 1 & $<0.33 \pm 0.16$ & $<21.27 \pm 0.23$ & $<0.71$ \\
2 & $<0.45 \pm 0.15$ & $<22.20 \pm 0.15$ & $<0.70$ \\
3 & $<0.20 \pm 0.11$ & $<21.37 \pm 0.28$ & $<0.69$ \\
\hline
\end{tabular}

Note. - Col. (1): Identification number assigned in this paper. Detections are followed by upper limits. Col. (2): Flux density (or upper limit) at $20 \mathrm{~cm}$ from FIRST (mJy). Col. (3): Corresponding radio power $\left(\mathrm{W} \mathrm{Hz}^{-1}\right)$. Col. (4): $R \equiv f_{6 \mathrm{~cm}} / f_{4400 \AA}$, assuming a spectral index of $\alpha_{\mathrm{r}}=0.46$ (radio; Ulvestad \& Ho 2001) and $\alpha_{\mathrm{o}}=0.44$ (optical; Vanden Berk et al. 2001), where $f_{\nu} \propto \nu^{-\alpha_{\mathrm{o}}}$. Table 6 is available in its entirety via the link to the machine-readable table above. The following is included only as a guide to content and presentation.

there is $\mathrm{a} \sim 2 \sigma$ detection in the inner five square pixels (where $\sigma$ is measured in a box off the center of the cut-out). We exclude these from our stacked images, although the answer changes negligibly when they are included. We do detect radio emission in each stacked image, shown as crossed squares in Figure 7 , also consistent with the general relation.

\subsection{Host Galaxies}

Naively, our expectation is that lower-mass BHs will be found, in general, in low-mass galaxies. However, the tight observed relations between BHs and galaxies pertain specifically to galaxy bulges. We are now in a position to test whether $\mathrm{BH}-$ bulge relations break down in low-mass galaxies. The spectra provide an estimate for the AGN luminosity (we prefer to use the $\mathrm{H} \alpha$ luminosity because of its robustness in the presence of significant galaxy contamination; Greene \& Ho 2005b), which we convert to magnitudes using SDSS filter functions and assuming a spectral shape of $\beta=-1.56$ (Vanden Berk et al. 2001). If we assume a flatter slope of $\beta=-1$, as suggested by $\mathrm{GH}$, the median magnitude difference is only $0.06 \mathrm{mag}$. In addition to the power law, we also include the observed strong emission lines $(\mathrm{H} \beta$, [O III], [N II], and $\mathrm{H} \alpha)$ in the calculation of the AGN luminosity (on average, line emission accounts for $\langle g\rangle \approx 0.08$ mag). Once we have removed our best estimate for the AGN luminosity from the SDSS Petrosian magnitudes, we calculate $K$ corrections using the routines described in Blanton \& Roweis (2007). The distribution of host galaxy luminosities thus derived has a median host galaxy luminosity of $\left\langle M_{g}\right\rangle=-19.3 \mathrm{mag}$ (with or without the $c$ sample) and is shown in Figure 8. With this method of AGN removal, we tend to underestimate the AGN luminosity due to fiber losses, and thereby

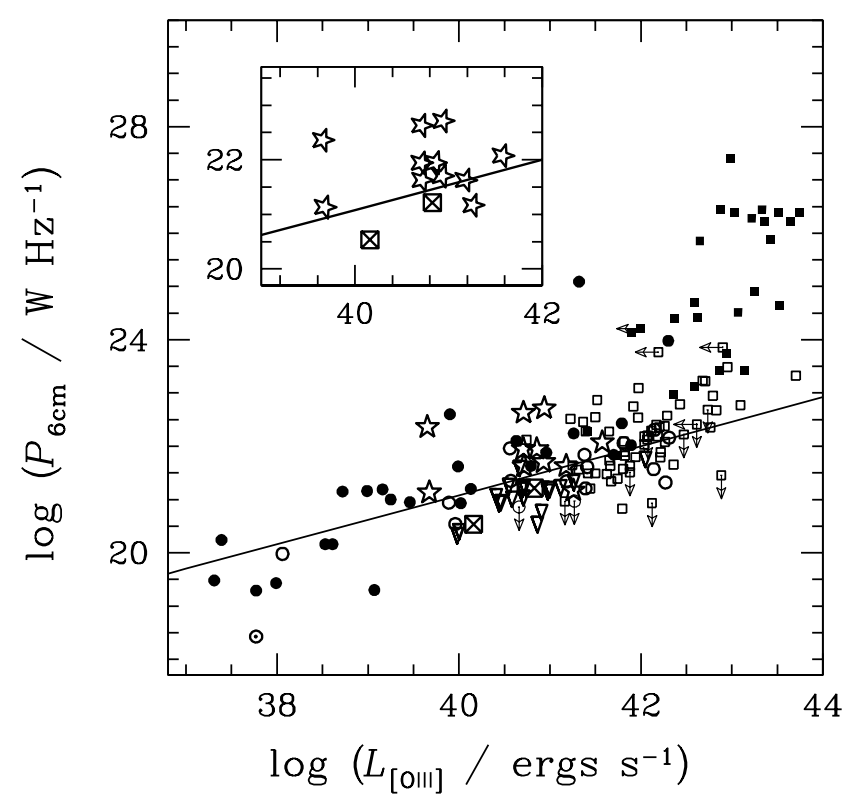

FIG. 7. - Adopted from Greene et al. 2006, this figure shows the relation between $L_{[\mathrm{OII}]}$ and radio power for a large sample of AGNs. Objects from this paper are shown as open stars, while stacked luminosities for undetected sources are shown as crossed squares and upper limits from the original GH sample are shown as open triangles. For clarity, our data are plotted alone in the inset. The solid line represents the fit from Ho \& Peng 2001 to radio-quiet Seyfert galaxies and Palomar-Green (PG; Schmidt \& Green 1983) quasars: $P_{6 \mathrm{~cm}}=(0.46 \pm 0.15) L_{[\mathrm{OIII}]}+(2.68 \pm 6.21)$. The Ho \& Peng sample is overplotted with filled (radio-loud sources) and open (radio-quiet sources) symbols. PG quasars are shown as squares, and Seyferts are shown as circles. NGC 4395, with $L_{[\mathrm{OIII}]}=5.8 \times 10^{37} \mathrm{ergs} \mathrm{s}^{-1}$ (adjusted to a distance of $4.2 \mathrm{Mpc}$; Thim et al. 2004) is highlighted as a semifilled circle.

(conservatively) overestimate the galaxy luminosity. As a lower bound on the galaxy luminosity, we use the SDSS point-spread function (PSF; unresolved) magnitude as an alternate estimate of the AGN luminosity, and then apply a $K$-correction to the corrected colors in the same manner. This method, on average, overestimates the AGN luminosity, and results in a median host galaxy luminosity of $\left\langle M_{g}\right\rangle=-19.0 \mathrm{mag}$ (Fig. 8, dotted histogram). The true host luminosities lie somewhere between these two bounds. For reference, Blanton et al. (2003) find a characteristic luminosity of $M_{g}^{*}=-20.1 \mathrm{mag}$ at $z=0.1$ (for our assumed cosmology), and thus our targets are $\sim 0.8-1.1 \mathrm{mag}$ fainter than $M_{g}^{*}$.

Morphological information is difficult to extract from the SDSS images alone, due to their limited depth and spatial resolution, but it is clear that BHs selected to have low masses are found in low-mass galaxies. However, if we restrict our attention to the 139 sources with $z<0.1$, we find $\sim 80$ clear disk galaxies (see GH for example SDSS images for the original sample). We can also calculate galaxy colors: $\langle g-r\rangle=0.7 \mathrm{mag}$ for the spectroscopic estimate of the AGN luminosity (0.6 mag for the PSF method). According to Fukugita et al. (1995), the host galaxies have the colors of typical Sab galaxies $(g-r=0.66$ mag). HST images of the GH sample indicate that there are actually two different populations of host galaxies. One is composed of late-type spiral galaxies, typically with strong bars. The other - the majority-group is comprised of compact spheroids, not unlike POX 52, the prototypical dwarf spheroidal galaxy hosting an AGN, which has red colors $(B-V=0.8$ or $g-r \approx 0.67 \mathrm{mag}$ ). We do not yet know if the spheroidal galaxies in the larger sample are red in general. 


\subsection{Stellar Populations and Ongoing Star Formation}

It is tempting to imagine that the constant mass ratio observed between BHs and bulges results from concurrent (or at least orchestrated) star formation and AGN activity. Indeed, various theories have postulated that AGN activity is responsible for truncating star formation (e.g., Springel et al. 2005). While characterizing the star formation rates of broadline AGNs is notoriously challenging, evidence for ongoing star formation has been seen in some cases (e.g., Canalizo \& Stockton 2001). With narrow-line objects the galaxy is easier to study directly, and Kauffmann et al. (2003) find a strong tendency for the most active objects in their sample of SDSS narrow-line AGNs to contain a significant post-starburst population. Ho (2005) use the [O II] doublet, which is intrinsically weak in high-ionization Seyfert galaxies, to find that star formation is suppressed in luminous AGNs, even when molecular gas is present (see also Kim et al. 2006); Ho speculates that this may be a concrete manifestation of AGN feedback. Our AGNs, while of low-mass and correspondingly low-luminosity, nevertheless have the highest growth rates of any local AGNs because of their high Eddington ratios (Greene \& Ho 2007b). Unfortunately, we do not have a large amount of information about the stellar populations, both because of the AGN dominance and the low typical signal-to-noise ratio of the spectra.

From the host galaxy colors, it is clear that recent star formation has occurred in some fraction of the galaxies. A more compelling argument for intermediate-age stellar populations comes from the strength of the higher-order Balmer lines apparent in the stacked spectra (Fig. 6). Balmer absorption is present in all Eddington ratio bins, but we cannot, from these data alone, quantify whether there is any correlation between AGN luminosity and strength of Balmer absorption. We are wary to measure stellar population indices for these objects without a clean measurement of the AGN luminosity, but the absorption-line spectra (particularly in panels $c$ and $d$ of Fig. 6) clearly contain a contribution from A stars.

Each of these clues only places constraints on star formation in the past $\sim 10^{9} \mathrm{yr}$. As always, it is far more challenging to find evidence for ongoing star formation, since the emission from the AGN itself generates nebular emission. The HST morphologies do suggest that star formation is ongoing at least in the spiral arms of the disk galaxies in the GH sample, but a more quantitative estimate is desirable. This was the motivation of Ho (2005) to use the [O II]/[O III] ratio as an indicator of concurrent star formation. Unfortunately, as we have seen above, we do not have a strong handle on the physical properties in the NLR, and therefore cannot uniquely interpret the observed spread in $[\mathrm{O} \mathrm{II}] /[\mathrm{O} \mathrm{III}]$ ratio. There is a mild correlation between the $[\mathrm{O} \mathrm{II}] /[\mathrm{O} \mathrm{III}]$ ratio and the $g-r$ color $\left(\rho=-0.3, P<10^{-4}\right.$; including or excluding the $c$ sample), which one might expect if the [O II] arises from current star formation. On the other hand, if the NLR conditions correlate with Hubble type (not an unreasonable possibility), and hence indirectly with galaxy color, a similar correlation may result. With these data alone, we cannot place strong constraints on the ongoing star formation in these systems, nor are constraints on star formation alone sufficient to implicate a causal connection with bulge growth. We would like to determine whether there is an excess of star formation in the AGN hosts as compared to inactive dwarf galaxies. A combination of high spatial resolution optical imaging with HST and broader spectral modeling of the host colors should be a first step toward this goal.
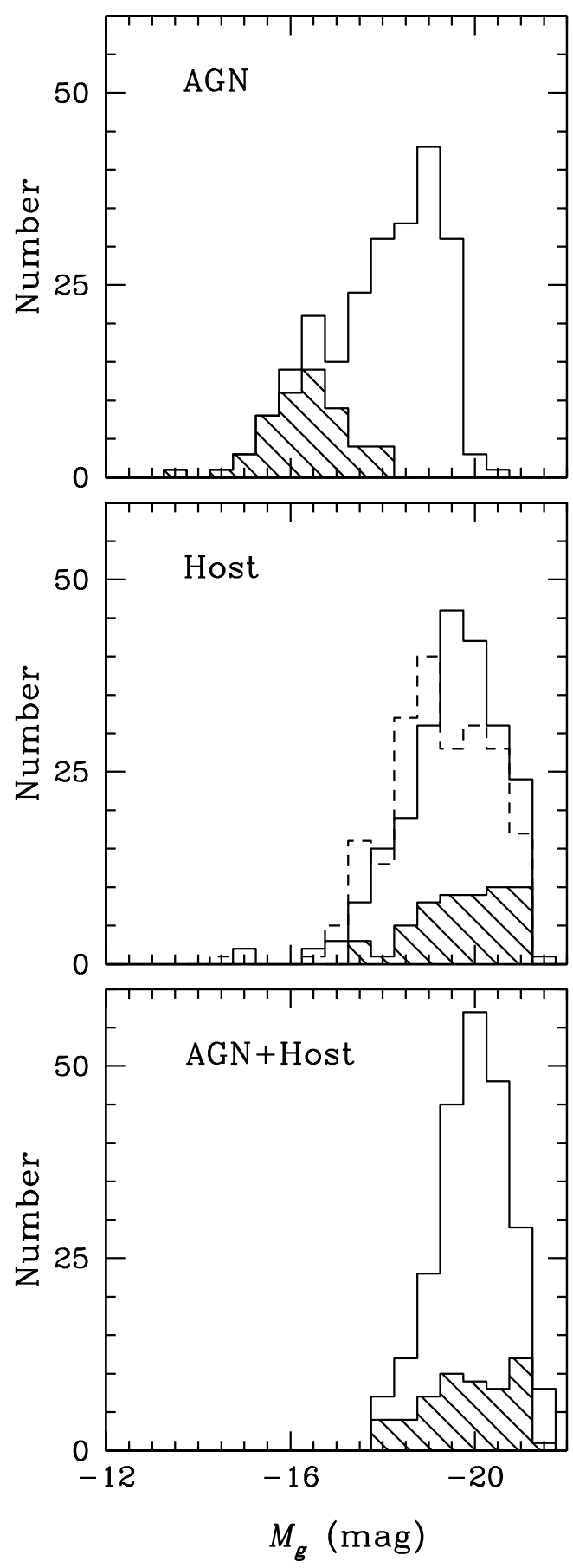

FIG. 8.- Distributions of $g$-band absolute magnitudes. The magnitudes pertain to the AGN alone (top), the host galaxy alone (middle), and the entire system (bottom). Filled histograms represent objects below the detection threshold defined in Greene \& Ho (2007b; the $c$ sample). The AGN contribution was estimated from $L_{\mathrm{H} \alpha}$, using the $L_{\mathrm{H} \alpha}-L_{5100}$ relation of Greene \& Ho $2005 \mathrm{~b}$, and assuming a spectral shape of $\beta=-1.56\left(f_{\lambda} \propto \lambda^{-\beta}\right.$; Vanden Berk et al. 2001). Alternatively, the AGN magnitude may be estimated from the SDSS PSF magnitude, which leads to the distribution shown in dotted lines in the middle panel.

\section{SUMMARY AND FUTURE WORK}

We have increased the sample of low-mass BHs in galactic nuclei by an order of magnitude (174+55 additional candidates). In general the sample properties are completely consistent with that of the original GH sample. The objects are radiating at $\sim$ one-third of their Eddington limit, and many of them have ROSAT counterparts. On the other hand, as expected, very 
few have radio detections in the FIRST survey.

Much like our previous sample, the current objects are found in sub- $L^{*}$ galaxies, suggesting that indeed low-mass BHs are found in low-mass stellar systems. The colors and composite spectral properties indicate that there are intermediate-age stellar populations, but we have no direct constraints on ongoing star formation. Although the SDSS imaging is neither deep enough nor of high enough angular resolution to determine the host galaxy structure, we do have relevant external data for the original GH sample. The bulge stellar velocity dispersions presented in Barth et al. (2005) show that the objects are consistent with the low-mass extrapolation of the $M_{\mathrm{BH}}-\sigma_{*}$ relation. There is a suggestion, from Greene \& Ho (2006), that the slope of the AGN $M_{\mathrm{BH}}-\sigma_{*}$ relation flattens somewhat at these low masses.

While the systems (at least to first order) obey the $M_{\mathrm{BH}}-\sigma_{*}$ relation, they do not necessarily contain classical bulges. NGC 4395 is an Sdm spiral galaxy (i.e., no bulge), while POX 52 is probably a dwarf spheroidal. As a result, the low-mass systems do not appear to obey the low-mass extrapolation of the $M_{\mathrm{BH}^{-}}$ - $L_{\text {bulge }}$ relation (e.g., Marconi \& Hunt 2003). The total galaxy luminosities are certainly larger than one would expect, based on their BH masses, and this appears to be true even in the absence of disk components (Barth et al. 2004; J. E. Greene et al. in preparation). Therefore even near their Eddington limits these BHs are not capable of providing as much energy per unit host galaxy mass as more massive systems. The mystery, then, is why they appear to obey the same $M_{\mathrm{BH}}-\sigma_{*}$ relation.

Along these lines, we should note that the revised radiusluminosity relation slope (Bentz et al. 2006) substantially increases the virial masses at low $\mathrm{BH}$ mass, which in turn increases the apparent flattening in the $M_{\mathrm{BH}}-\sigma_{*}$ relation slope at low mass previously reported by Greene \& Ho (2006). More insight should be gained into the reality of this slope change once we have measured $\sigma_{*}$ for the present sample. Ultimately, we wish to elucidate the degree to which the slope and (crucially) intrinsic scatter of the $M_{\mathrm{BH}}-\sigma_{*}$ relation has any mass dependence (e.g., Robertson et al. 2006; Greene \& Ho 2006).
Indeed, there are a number of interesting applications to pursue with the improved statistics afforded by this new, larger sample. We plan to examine the environments and clustering properties of these objects, and see how they compare to inactive galaxies of similar mass and color. At the same time, we would like to constrain the stellar populations of the host galaxies in a more quantitative fashion. For the GH sample, at least, this will be enabled by a combination of HST imaging and Spitzer IRS spectra. Most immediately, we will use the HST imaging, in combination with stellar velocity dispersion measurements, to place the GH sample on the fundamental plane. In combination, we will use these data to examine differences in the evolution of low-mass systems as compared to supermassive BHs in massive bulges.

We acknowledge the useful suggestions of the anonymous referee that improved this work significantly. We thank Michael Blanton for making his $K$-correction code publicly available, and Brent Groves for kindly providing his metallicity models prior to publication. We thank the entire SDSS team for providing the fantastic data products that made this work possible. Support for J. E. G. was provided by NASA through Hubble Fellowship grant HF-01196 awarded by the Space Telescope Science Institute, which is operated by the Association of Universities for Research in Astronomy, Inc., for NASA, under contract NAS 5-26555. L. C. H. acknowledges support by the Carnegie Institution of Washington and by NASA grant SAO 06700600. Funding for the SDSS has been provided by the Alfred P. Sloan Foundation, the Participating Institutions, the National Science Foundation, the U.S. Department of Energy, the National Aeronautics and Space Administration, the Japanese Monbukagakusho, the Max Planck Society, and the Higher Education Funding Council for England. The SDSS web site is http://www.sdss.org/. This research has made use of data obtained from the High Energy Astrophysics Science Archive Research Center (HEASARC), provided by NASA's Goddard Space Flight Center.

\section{APPENDIX}

\section{VIRIAL BLACK HOLE MASS MEASUREMENTS}

The virial technique is quite indirect, and its calibration is a matter of ongoing research. Therefore, it is worth describing our methodology in some detail. In particular, we discuss the potential systematic errors incurred by uncertainties in the slope of the radius-luminosity relation on the one hand and the derivation of robust gas velocity dispersions on the other.

At the present time, the radius-luminosity relation is calibrated using the $\sim 30$ objects with BLR radii determined from reverberation mapping. Bentz et al. have used HST to determine the true AGN luminosity, as free as possible of host-galaxy contamination. Their quoted radius-luminosity relation slope is significantly shallower than that of Kaspi et al. (2005). It is worth keeping in mind the important caveat that the luminosities of the objects in that study barely overlap with those in this work, and most are significantly higher than those considered here. While programs to measure BLR radii for lower-luminosity sources are underway, at the moment we have no choice but to adopt the results for the more luminous sources.

As described in detail in Greene \& Ho (2005b), the line luminosity provides a more robust measure of the AGN luminosity in the presence of host galaxy contamination (or contamination from non-thermal jet emission). Therefore we have used the $L_{5100}-L_{\mathrm{H} \alpha}$ relation from Greene \& Ho (2005b) in combination with the revised radius-luminosity relation from Bentz et al. (and uncertainties from M. Bentz, private communication) to derive a final $\mathrm{BH}$ mass estimator:

$$
M_{\mathrm{BH}}=\left(3.0_{-0.5}^{+0.6}\right) \times 10^{6}\left(\frac{L_{\mathrm{H} \alpha}}{10^{42} \mathrm{erg} \mathrm{s}^{-1}}\right)^{0.45 \pm 0.03}\left(\frac{\mathrm{FWHM}_{\mathrm{H} \alpha}}{10^{3} \mathrm{~km} \mathrm{~s}^{-1}}\right)^{2.06 \pm 0.06} M_{\odot}
$$

where we assume a spherical BLR $(f=0.75)$. Here we are using the FWHM of H $\alpha$ as a measure of the gas velocity dispersion. In principle the width of the broad emission lines represents the gas kinematics, but in practice it is unclear how to derive the most robust velocity dispersion measure. It has long been clear that the broad line shape depends systematically on other AGN properties 
including line width (or BH mass) and luminosity (e.g., Sulentic et al. 2000); in general, narrower lines have more Lorentzian shapes (e.g., Véron-Cetty et al. 2001). If the geometry and kinematics of the BLR change with Eddington ratio (as suggested by these observations) then the assumption of a single geometrical factor $f$ breaks down. In other words, the measured virial mass for a given BH may vary as changes in the Eddington ratio lead to changes in the BLR kinematics and thereby $f$. An extreme example of this effect is reported for AGNs with double-peaked Balmer lines (Bian et al. 2007).

Without independent information it is impossible to determine $f$ as a function of $L_{\mathrm{bol}} / L_{\mathrm{Edd}}$. Luckily, the $M_{\mathrm{BH}}-\sigma_{*}$ relation provides an independent handle on the BH mass. Especially for the relatively low-luminosity AGNs considered at low redshift, it is safe to assume that the systems intrinsically obey the $M_{\mathrm{BH}}-\sigma_{*}$ relation, and adopt the $M_{\mathrm{BH}}-\sigma_{*}$-derived mass as the true $\mathrm{BH}$ mass. Both Onken et al. (2004) and Greene \& Ho (2006) find evidence for an average offset However, as argued above, we expect that $f$ is not a constant, but rather depends on $L_{\mathrm{bol}} / L_{\mathrm{Edd}}$. While Greene \& Ho (2006) looked for evidence of a trend between $f$ and $L_{\mathrm{bol}} / L_{\mathrm{Edd}}$, their dynamic range in both $\mathrm{BH}$ mass and luminosity was too limited to draw robust conclusions.

Collin et al. (2006) perform an exhaustive study of the relation between line shape and virial mass. They use the Onken et al. sample of 14 AGNs with both reverberation-mapped masses and $\sigma_{*}$ measurements to investigate the merits of different measures of the line width. Specifically, they compare virial BH masses estimated from the FWHM and the luminosity-weighted second moment of the velocity profile $(\sigma)$ with the $\sigma_{*}$-derived BH mass. As described above, they find that narrower lines are peaky (small values of FWHM $/ \sigma$ ) while broader lines are boxy (large values of FWHM $/ \sigma$ ). As a result, the inferred $f$ may depend not only on whether FWHM or $\sigma$ is used to measure line width, but also on the line shape. Using the comparison between virial mass and $\sigma_{*}$, they derive a different value of $f$ for each subset of objects ( 9 peaky and 5 boxy) using both FWHM and $\sigma$. They claim that while $f$ is constant across line samples when $\sigma$ is used as the velocity dispersion indicator, the FWHM provides a biased measure of the virial mass because $f$ is smaller for the boxy subset. However, investigation of their table reveals that the values of $f$ derived from FWHM measurements are also consistent (within the quoted errors) with a constant value. Although the result may very well be real, it is certainly not statistically significant at this time.

Moreover, we question the premise of Collin et al. that the best measure of line width is the one that minimizes changes in $f$. Rather, since the BLR geometry really does change with $L_{\mathrm{bol}} / L_{\mathrm{Edd}}$, one should calibrate different $f$ values for different average line shapes, when sufficient statistics exist to do so in a robust fashion. Collin et al. present an additional piece of information that we believe favors use of the FWHM over $\sigma$. Because they are using reverberation-mapped AGNs, many epochs of observations are available. One may derive a so-called rms spectrum that isolates the variable part of the line. As argued in, e.g., Peterson et al. (2004), it is preferable to measure the width of the variable component of the line, which hopefully reflects the kinematics of gas at the measured radii. As it turns out, the FWHM measured from the mean and rms spectra are more self-consistent than the respective $\sigma$ measurements. There appears to be a very broad, non-variable component of the line which biases $\sigma$ more strongly than FWHM (perhaps an optically thin component; e.g., Shields et al. 1995). While the very broad component cannot be removed in single-epoch observations, at least the FWHM is less biased by it. Finally, it is quite clear from Figure 1 of Collin et al. that the scatter in $\sigma$ measurements is considerably larger than in the FWHM. The same result was reported by Greene \& Ho (2006).

Based on all of these considerations, the following choices were made in calculating the BH masses presented herein:

- We use the radius-luminosity relation reported by Bentz et al. (2006). Note that this differs from Greene \& Ho (2007b).

- We use the FWHM to determine the gas velocity dispersion.

- Because we believe $f$ is not a constant, but rather depends on $L_{\mathrm{bol}} / L_{\mathrm{Edd}}$, and in order to facilitate comparison with the literature, we do not apply an $f$ correction but use the fiducial assumption of a spherical BLR $(f=0.75$; e.g., Netzer 1990).

Obviously, virial masses remain highly uncertain, and potentially systematically biased. In addition to the many uncertainties highlighted above, it is not yet clear the degree to which the BLR is a flattened structure, which means that inclination effects cannot be properly accounted for, even in a statistical sense. We are hopeful that an increased sample of reverberation-mapped sources (e.g., Kollatschny 2003), combined with an increase in the number of measured $\sigma_{*}$ measurements in AGNs, will decrease the outstanding systematic errors in these masses in the near future.

\footnotetext{
${ }^{4}$ Note that the Greene \& Ho measurement, while including many more AGNs, is still dominated by the reverberation-mapped sources, which have considerably smaller systematic errors in their virial BH mass estimates.
} 


\section{REFERENCES}

Adelman-McCarthy, J. K., et al. 2006, ApJS, 162, 38

Baldwin, J. A., Phillips, M. M., \& Terlevich, R. 1981, PASP, 93, 5

Barth, A. J., Greene, J. E., \& Ho, L. C. 2005, ApJ, 619, L151

Barth, A. J., Ho, L. C., Rutledge, R. E., \& Sargent, W. L. W. 2004, ApJ, 607, 90

Becker, R. H., White, R. L., \& Helfand, D. J. 1995, ApJ, 450, 559

Bennert, N., Falcke, H., Schulz, H., Wilson, A. S., \& Wills, B. J. 2002, ApJ, 574, L105

Bentz, M. C., Peterson, B. M., Pogge, R. W., Vestergaard, M., \& Onken, C. A. 2006, ApJ, 644, 133

Best, P. N., Kauffmann, G., Heckman, T. M., Brinchmann, J., Charlot, S., Ivezić, Ž., \& White, S. D. M. 2005, MNRAS, 362, 25

Bian, W.-H., Chen, Y.-M., Gu, Q.-S., \& Wang, J.-M. 2007, ApJ, accepted (arXiv:0706.2473)

Blanton, M. R., et al. 2003, ApJ, 592, 819

Blanton, M. R., \& Roweis, S. 2007, AJ, 133, 734

Boller, Th., Brandt, W. N., \& Fink, H. 1996, A\&A, 305, 53

Boroson, T. A. 2002, ApJ, 565, 78

Boroson, T. A., \& Green, R. F. 1992, ApJS, 80, 109

Canalizo, G., \& Stockton, A. 2001, ApJ, 555, 719

Collin, S., Kawaguchi, T., Peterson, B. M., \& Vestergaard, M. 2006, A\&A, 456, 75

Condon, J. J. 1989, ApJ, 338, 13

Dong, X., et al. 2007a, ApJ, 657, 700

Dong, X., Wang, T., Yuan, W., Zhou, H., Shan, H., Wang, H., Lu, H., \& Zhang, K. 2007b, in The Central Engine of Active Galactic Nuclei, ed. L. C. Ho \& J.-M. Wang (San Francisco: ASP), in press

Eisenhauer, F., et al. 2005, ApJ, 628, 246

Fabbiano, G. 1989, ARA\&A, 27, 87

Favata, M., Hughes, S. A., \& Holz, D. E. 2004, ApJ, 607, L5

Feigelson, E. D., \& Nelson, P. I. 1985, ApJ, 293, 192

Ferrarese, L., \& Merritt, D. 2000, ApJ, 539, L9

Ferrarese, L., Pogge, R. W., Peterson, B. M., Merritt, D., Wandel, A.,\& Joseph, C. L. 2001, ApJ, 555, L79

Filippenko, A. V., \& Halpern, J. P. 1984, ApJ, 285, 458

Filippenko, A. V., \& Ho, L. C. 2003, ApJ, 588, L13

Filippenko, A. V., \& Sargent, W. L. W. 1989, ApJ, 342, L11

Fine, S., et al. 2006, MNRAS, 373, 613

Francis, P. J., Hewett, P. C., Foltz, C. B., Chaffee, F. H., Weymann, R. J., \& Morris, S. L. 1991, ApJ, 373, 465

Fukugita, M., Shimasaku, K., \& Ichikawa, T. 1995, PASP, 107, 945

Gaskell, C. M., \& Ferland, G. J. 1984, PASP, 96, 393

Gebhardt, K., et al. 2000a, ApJ, 539, L13

- 2000b, ApJ, 543, L5 2001, AJ, 122, 2469

Gebhardt, K., Rich, R. M., \& Ho, L. C. 2002, ApJ, 578, L41

. 2005, ApJ, 634, 1093

Ghez, A. M., Salim, S., Hornstein, S. D., Tanner, A., Lu, J. R., Morris, M., Becklin, E. E., \& Duchêne, G. 2005, ApJ, 620, 744

Greene, J. E., \& Ho, L. C. 2004, ApJ, 610, 722

-. 2005a, ApJ, 627, 721

- 2005b, ApJ, 630, 122

- 2006, ApJ, 641, L21

- 2007a, ApJ, 656, 84

2007b, ApJ, accepted (astroph/0705.0020)

Greene, J. E., Ho, L. C., \& Ulvestad, J. S. 2006, ApJ, 636, 56

Groves, B. A., Dopita, M. A., \& Sutherland, R. S. 2004a, ApJS, 153, 9

. 2004b, ApJS, 153, 75

Groves, B. A., Heckman, T. M., \& Kauffmann, G. 2006, MNRAS, 371, 1559

Halpern, J. P., \& Steiner, J. E. 1983, ApJ, 269, L37

Hao, L., et al. 2005, AJ, 129, 1783

Heckman, T. M. 1980, A\&A, 87, 152

Ho, L. C. 2005, ApJ, 629, 680

. 2004, in Carnegie Observatories Astrophysics Series, Vol. 1: Coevolution of Black Holes and Galaxies, ed. L. C. Ho (Cambridge: Cambridge Univ. Press), 292

Ho, L. C., Filippenko, A. V., \& Sargent, W. L. W. 1997a, ApJS, 112, 315
- 1997b, ApJ, 487, 568

Ho, L. C., Filippenko, A. V., Sargent, W. L. W., \& Peng, C. Y. 1997c, ApJS, 112,391

Ho, L. C. \& Peng, C. Y. 2001, ApJ, 555, 650

Ho, L. C., Shields, J. C., \& Filippenko, A. V. 1993, ApJ, 410, 567

Hughes, S. A. 2002, MNRAS, 331, 805

Kaspi, S., Maoz, D., Netzer, H., Peterson, B. M., Vestergaard, M., \& Jannuzi, B. T. 2005, ApJ, 629, 61

Kauffmann, G., et al. 2003, MNRAS, 346, 1055

Kim, M., Ho, L. C., \& Im, M. 2006, ApJ, 642, 702

Kollatschny, W. 2003, A\&A, 407, 461

Kraemer, S. B., Ho, L. C., Crenshaw, D. M., Shields, J. C., \& Filippenko, A. V. 1999, ApJ, 520, 564

Krolik, J. H. 2001, ApJ, 551, 72

Kunth, D., Sargent, W. L. W., \& Bothun, G. D. 1987, AJ, 93, 29

Leighly, K. M. 1999, ApJS, 125, 297

Marconi, A., \& Hunt, L. K. 2003, ApJ, 589, L21

McClintock, J. E., \& Remillard, R. A. 2006, in Compact stellar X-ray sources. Edited by Walter Lewin \& Michiel van der Klis. Cambridge Astrophysics Series, No. 39. Cambridge, UK: Cambridge University Press

McLure, R. J., \& Dunlop, J. S. 2004, MNRAS, 352, 1390

Merritt, D., Milosavljević, M., Favata, M., Hughes, S. A., \& Holz, D. E. 2004, ApJ, 607, L9

Meylan, G., Sarajedini, A., Jablonka, P., Djorgovski, S. G., Bridges, T., \& Rich, R. M. 2001, AJ, 122, 830

Mukai, K. 1993, Legacy, vol. 3, 3, 21

Nelson, C. H., Green, R. F., Bower, G., Gebhardt, K., \& Weistrop, D. 2004, ApJ, 615, 652

Netzer, H. 1990, in Active Galactic Nuclei, ed. R. D. Blandford, H. Netzer, L. Woltjer, T. Courvoisier, \& M. Mayor, (Berlin: Springer), 57

Netzer, H., Mainieri, V., Rosati, P., \& Trakhtenbrot, B. 2006, A\&A, 453, 525

Netzer, H., Shemmer, O., Maiolino, R., Oliva, E., Croom, S., Corbett, E., \& di Fabrizio, L. 2004, ApJ, 614, 558

Onken, C. A., Ferrarese, L., Merritt, D., Peterson, B. M., Pogge, R. W., Vestergaard, M., \& Wandel, A. 2004, ApJ, 615, 645

Osterbrock, D. E., \& Pogge, R. W. 1985, ApJ, 297, 166

Peterson, B. M., et al. 2005, ApJ, 632, 799

- 2004, ApJ, 613, 682

Pooley, D., \& Rappaport, S. 2006, ApJ, 644, L45

Pounds, K. A., Done, C., \& Osborne, J. P. 1995, MNRAS, 277, L5

Robertson, B., Hernquist, L., Cox, T. J., Di Matteo, T., Hopkins, P. F., Martini, P., \& Springel, V. 2006, ApJ, 641, 90

Schmidt, M., \& Green, R. F. 1983, ApJ, 269, 352

Schmitt, H. R., Donley, J. L., Antonucci, R. R. J., Hutchings, J. B., Kinney, A. L., \& Pringle, J. E. 2003, ApJ, 597, 768

Shields, J. C., Ferland, G. J., \& Peterson, B. M. 1995, ApJ, 441, 507

Spergel, D. N., et al. 2003, ApJS, 148, 175

Springel, V., Di Matteo, T., \& Hernquist, L. 2005, ApJ, 620, L79

Steffen, A. T., Strateva, I., Brandt, W. N., Alexander, D. M., Koekemoer, A. M., Lehmer, B. D., Schneider, D. P., \& Vignali, C. 2006, AJ, 131, 2826

Strateva, I. V., Brandt, W. N., Schneider, D. P., Vanden Berk, D. G., \& Vignali, C. 2005, AJ, 130, 387

Sulentic, J. W., Zwitter, T., Marziani, P., \& Dultzin-Hacyan, D. 2000, ApJ, 536, L5

Thim, F., Hoessel, J. G., Saha, A., Claver, J., Dolphin, A., \& Tammann, G. A. 2004, AJ, 127, 2322

Tremaine, S., et al. 2002, ApJ, 574, 740

Tremonti, C. A., et al. 2004, ApJ, 613, 898

Ulvestad, J. S., Greene, J. E., \& Ho, L. C. 2007, ApJ, 661, L151

Ulvestad, J. S., \& Ho, L. C. 2001, ApJ, 558, 561

Valluri, M., Ferrarese, L., Merritt, D., \& Joseph, C. L. 2005, ApJ, 628, 137

Vanden Berk, D. E., et al. 2001, AJ, 122, 549

Veilleux, S., \& Osterbrock, D. E. 1987, ApJS, 63, 295

Véron-Cetty, M.-P., Véron, P., \& Gonçalves, A. C. 2001, A\&A, 372, 730

Voges, W., et al. 1999, A\&A, 349, 389

Williams, R. J., Mathur, S., \& Pogge, R. W. 2004, ApJ, 610, 737

York, D. G., et al., 2000, AJ, 120, 1579 